\title{
A Comparative Analysis of Multiple Fractional Solutions of Generalized Couette Flow of Couple Stress Fluid in Channel
}

\section{MUHAMMAD ARIF}

King Mongkut's University of Technology Thonburi

POOM KUMAM ( $\nabla$ poom.kum@kmutt.ac.th)

China Medical University Hospital

\section{WIYADA KUMAM}

Rajamangala University of Technology

\section{Research Article}

Keywords: Generalized Couette flow, Couple stress fluid, Caputo, Caputo-Fabrizio, Atangana-Baleanu, Joint applications of Laplace and Fourier transform

Posted Date: March 4th, 2021

DOl: https://doi.org/10.21203/rs.3.rs-275575/v1

License: (9) This work is licensed under a Creative Commons Attribution 4.0 International License. Read Full License 


\title{
A Comparative Analysis of Multiple Fractional Solutions of Generalized Couette Flow of Couple Stress Fluid in Channel
}

\author{
MUHAMMAD ARIF ${ }^{1}$, POOM KUMAM ${ }^{*, 1,2}$, AND WIYADA KUMAM ${ }^{3}$ \\ ${ }^{1}$ Center of Excellence in Theoretical and Computational Science (TaCS-CoE) \& KMUTTFixed Point Research \\ Laboratory, Room SCL 802 Fixed Point Laboratory, Science Laboratory Building, Departments of \\ Mathematics, Faculty of Science, King Mongkut's University of Technology Thonburi (KMUTT), \\ 126 Pracha-Uthit Road, Bang Mod, Thrung Khru, Bangkok 10140, Thailand \\ ${ }^{2}$ Department of Medical Research, China Medical University Hospital, China Medical University, Taichung 40402, \\ Taiwan \\ ${ }^{3}$ Program in Applied Statistics, Department of Mathematics and Computer Science, Faculty of Science and \\ Technology, Rajamangala University of Technology Thanyaburi, Thanyaburi, Pathumthani 12110, Thailand \\ *Corresponding author: Poom Kumam (e-mail: poom.kum@kmutt.ac.th ).
}

\begin{abstract}
This study provides the exact solutions of couple stress fluid (CSF) in channel. The Couple stress fluid is bounded by two plates in which the lower plate is moving with constant velocity Uo and the upper plate is fixed. The influence of external pressure gradient is considered on the CSF fluid. To transform classical CSF model we have introduced three approaches of fractional derivatives, (a) Caputo (b) Caputo-Fabrizio (CF) and (c) Atangana-Baleanu $(\mathrm{AB})$ fractional definitions. The exact solutions have been obtained using the Laplace and finite Fourier sine transforms jointly. Furthermore, the effect of different fractional derivatives is compared and the results are shown in graphs. Moreover, parameters which effect the CSF motion are discussed in the graph. The most important out come from the given study is the comparison of Caputo, Caputo-Fabrizio and Atangana-Baleanu fractional models with classical CSF model. From the comparison it can be noticed that Atangana-Baleanu fractional model discuss the dynamics of the couple stress fluid with good memory effect as compared to Caputo and Caputo-Fabrizio fractional operators. The CSF model can be reduced to Newtonian fluid as limiting case and also investigated solutions in the absence of external pressure. Finally, Skin friction are evaluated for lower and upper plates and presented the obtained results in tabular form.
\end{abstract}

Key Words: Generalized Couette flow; Couple stress fluid; Caputo; Caputo-Fabrizio; Atangana-Baleanu; Joint applications of Laplace and Fourier transform.

\section{Introduction}

Couple stress fluid is famous fluid in non-Newtonian fluids having enormous applications in fluid dynamics. The concept of CSF theory, was first given by Stokes [1], considers couple stresses in addition to the classical Cauchy stress. Couple stress fluid have many real world problems in engineering and biological sciences.

CSF models are very famous in non-Newtonian fluids. Due to this popularity of CSF many researchers investigated in different situations. The CSF model have been considered by Sha et al. [2] in this study they discussed couple stress 3D using stretched surface. Nagaraju et al. [3] discussed Entropy taking couple stress fluid in cylinder. Kumar et al. [4] also discussed unsteady couple stress nanofluid flow with Ohmic dissipation. Khan et al. [5] analyzed the swirling of couple stress fluid using a rotating disk. Hayat et al. [6] studied CSF flow with Cattaneo-Christov and double diffusion. Ahmad et al. [7] studied the CSF flow in inclined rotating channel. Beg et al. [8] calculated mathematical model of CSF biofluid in an inclined plane. Yaday et al. [9] discussed the analysis of internal heat generation in CSF in a saturated porous media. Furthermore, the analytical solutions of CSF flow in 
concentric cylinders was investigated by Devakar et al. [10]. Naeem et al. [11] studied some classes of CSF flow. Mekheimer [12] analyzed the CSF flow in an annulus for endoscopic applications.

The idea of fractional calculus is getting more attention from last three decades. This concept was originated in 1695. After that many real world problems and physical phenomenon were explained by researcher's using some suitable fractional order derivatives definitions. The use of fractional calculus is not only limited to engineering but it has many applications in biological sciences and some other field of sciences. Initially, researchers attempts many calculations and find some unique applications of fractional derivatives which are explained by Oldham and Spanier [13]. The applications of fractional derivatives are not only limited to engineering problmes but it has many industrial applications which are discussed in [14-16]. Due to the uses and novel applications of fractional derivatives many definitions proposed related to fractional derivative. In these definitions, the mostly used is Reimann-Liouville definition. There are two shortcomings in the Reimann-Liouville definition, firstly the derivative of some constant not equal to zero and secondly, the Laplace transform have some terms which have no physical meaning. After Reimann-Liouville Caputo develop a new fractional order derivative which overcome the shortcomings of Reimann-Liouville definition, but still there is a problem of singularity in its kernel.

There are many fractional order derivatives which have been used for different real world problems and scientific reason. The present article focused to highlight the most recent definitons of fractional derivatives. After Reimann-Liouville definition Caputo [17] developed a fractional derivatives which already used in many problems. After Caputo in 2015 Caputo and Fabrizio developed a fractional definition namely, Caputo-Fabrizio [18] fractional order derivatives. Recently, Atangana-Baleanu [19] introduced a modified definiton which have many uses in science and engineering. The comparison of time fractional derivative of $\mathrm{AB}$ and $\mathrm{CF}$ developed by Arif et al. [20], to investigate CSF model for generalized Couette flow. Akhtar [21] studied couple stress fluid usind different fractional apprpoches.

Recently, three fractional order derivatives have been developed which are applying in different real world problems and find the accurate solutions of those problems. In this study we collect some combined applications of these fractional derivatives. Motivated from the above mentioned literature this paper presents the comparison among these fractional order derivatives on the CSF fluid model in channel. The classical CSF model is transformed by applying first Caputo, Caputo-Fabrizio and then Atangana-Baleanu fractional approaches. The exact solutions have been recovered by applying the Fourier and Laplace transforms. The obtain solutions for Caputo, $\mathrm{CF}$ and $\mathrm{AB}$ have been compared with the classical couple stress model. From the comparison we found that AB fractional model discuss the dynamics of the couple stress fluid with good memory effect as compared to Caputo and CaputoFabrizio fractional operators.

\section{Mathematical Modeling And Solution Of The Problem}

This section provides mathematical modeling and exact solutions of CSF in channel. The fluid is taken in channel bounded by two plates the distance between them is taken $h$. The governing equations of the present study are given below [22]:

$$
\begin{gathered}
\nabla \cdot \vec{V}=0 \\
\rho \frac{\partial \vec{V}}{\partial t}=-\nabla p-\mu \nabla \times \nabla \times \vec{V}-\chi \nabla \times \nabla \times \nabla \times \nabla \times \vec{V}+\rho \overrightarrow{\mathrm{b}_{1}},
\end{gathered}
$$

Unidirectional CSF is taken in the present paper. Therefeore, $\vec{V}$ shows the velocity field in the x-direction. The components form of CSF can be written as [23]. 


$$
\rho \frac{\partial u(y, t)}{\partial t}=-\frac{\partial p}{\partial x}+\mu \frac{\partial^{2} u(y, t)}{\partial y^{2}}-\chi \frac{\partial^{4} u(y, t)}{\partial y^{4}}
$$

\subsection{The Generalized Couette Flow}

The present article describes the fluid motion between the two plates in the presence of pressure. The pressure is applied along the direction of the fluid which accelerates the fluid motion. The physical geometry of the given model is shown in Figure 1.

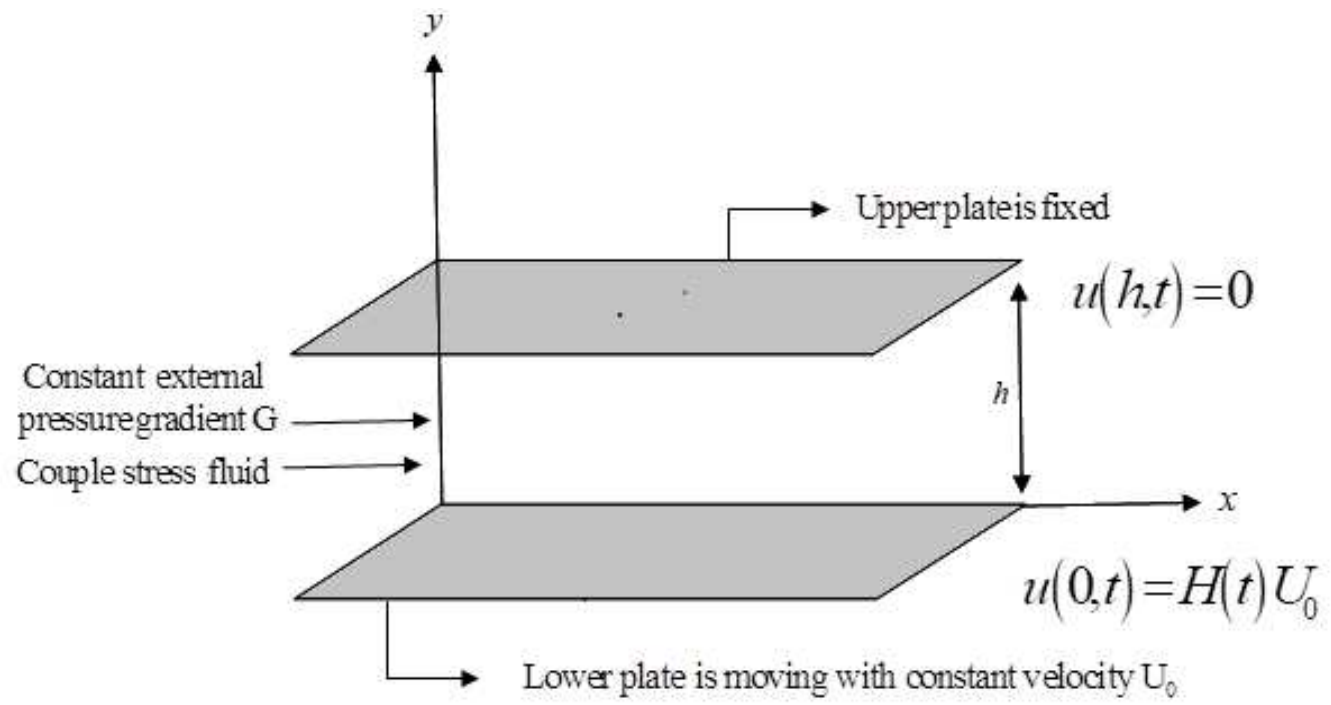

Figure.1: Physical sketch of the problem

The equations which govern the flow can be written as [24]:

$$
\rho u_{t}=G^{*}+\mu u_{y y}-\chi u_{y y y y},
$$

The IC's and BC's are given as:

$$
\begin{aligned}
& 0 \leq y \leq h \text { and } t=0, \quad u(y, t)=0, \\
& y=0 \text { and } t>0, u(y, t)=H(t) U_{0}, \\
& y=h \text { and } t>0, \quad u(y, t)=0, \\
& y=0 \text { and } \mathrm{y}=h, \quad u_{y y}(y, t)=0 .
\end{aligned}
$$

In order to get the dimensionless governing equations using the following non-dimensional quantities:

$$
\xi=\frac{y}{h} ; \quad \eta=\frac{u}{U_{0}} ; \quad \tau=\frac{U_{0} t}{h} .
$$


The dimensionless initial and boundary value problem is given below:

$$
\lambda \eta_{\xi \xi \xi \xi}-\eta_{\xi \xi}-G+\operatorname{Re} \eta_{\tau}=0
$$

The following are the dimensionless IC's and BC's:

$$
\left.\begin{array}{c}
\eta(\xi, \tau)=0, \quad \text { for } 0 \leq \xi \leq 1 \text { and } \tau=0, \\
\eta(\xi, \tau)=1, \quad \text { for } \xi=0 \text { and } \tau>0, \\
\eta(\xi, \tau)=0, \quad \text { for } \xi=1 \text { and } \tau>0, \\
\frac{\partial^{2} \eta(\xi, \tau)}{\partial \xi^{2}}=0, \text { at } \xi=0 \text { and } \xi=1 .
\end{array}\right\}
$$

here $\operatorname{Re}$ and $G$ is the Reynolds number and pressure $\lambda$ is particles size effect of couple stress fluid.

\section{Exact Solution}

In the present article the three fractional operator like, Caputo, $\mathrm{CF}$ and $\mathrm{AB}$ definitions are applied to simple CSF model in oreder to compare the results of these fractional approaches. Solutions of CSF model with Caputo fractional approach is given in section 3.1, solutiions of CSF model with Caputo-Fabrizio is given in 3.2 and solutions of CSF model with Atangana-Baleanu fractional derivative is given in subsection 3.3.

\subsection{Exact Solutions Using Caputo Definition}

Applying Caputo fractional definition to CSF model we get the following frictional model:

$$
{ }^{C} \mathbf{D}_{\tau}^{\gamma} \operatorname{Re\eta }(\xi, \tau)=G+\eta_{\xi \xi}-\lambda \eta_{\xi \xi \xi \xi},
$$

where ${ }^{C} \mathbf{D}_{\tau}^{\gamma}$ (.) is the Caputo definition of order $\gamma$ which is defined as $[25,26]$.

$$
{ }^{C} \mathbf{D}_{\tau}^{\gamma} \eta(\tau)=\frac{1}{\Gamma(1-\gamma)} \int_{0}^{\tau}(\tau-t)^{-\gamma} \eta^{\prime}(t) d t, 0 \leq \gamma<1
$$

Applying first the Laplace and then Fourier sine transforms to Eq. (8) using the conditions from Eq. (7), our transformed solution is given as:

$$
\operatorname{Re} p^{\gamma} \bar{\eta}_{s}(n, p)=\frac{G\left(1-(-1)^{n}\right)}{p \sigma_{n}}+\frac{\sigma_{n}}{p}-\sigma_{n}^{2} \bar{\eta}_{s}(n, p)+\lambda \frac{\sigma_{n}^{3}}{p}-\lambda \sigma_{n}^{4} \bar{\eta}_{s}(n, p)
$$

from above we can write as: 


$$
\bar{\eta}_{s}(n, p)=\left(\frac{G\left(1-(-1)^{n}\right)+\sigma_{n}^{2}+\lambda \sigma_{n}^{4}}{p \sigma_{n}\left(\operatorname{Re} p^{\gamma}+\sigma_{n}^{2}+\lambda \sigma_{n}^{4}\right)}\right),
$$

After some mathematical calculation our solution is given as:

$$
\bar{\eta}_{s}(n, p)=\left(\frac{G\left(1-(-1)^{n}\right)+\sigma_{n}^{2}+\lambda \sigma_{n}^{4}}{\sigma_{n} \operatorname{Re}}\right) \times\left(\frac{1}{H_{1} p}+\frac{1}{p^{1-\gamma}\left(p^{\gamma}+H_{1}\right)}\right)
$$

Applying both the inverse Laplace and Fourier transforms our solution is given in the following form:

$\eta(\xi, \tau)=\left(1-\left(\frac{2-G d^{2}}{2 d}\right) \xi-G-\frac{G \xi^{2}}{2}+G\left\{\frac{\cosh \left(\frac{d-2 \xi}{2}\right)}{\cosh \left(\frac{d}{2}\right)}\right\}\right)+\frac{2}{h} \sum_{n=1}^{\infty} \chi_{0} h(t) * E_{\gamma}\left(-H_{1}, \tau\right) \sin \left(\sigma_{n} \xi\right)$

where $H_{1}=\frac{\sigma_{n}^{2}+\lambda \sigma_{n}^{4}}{\operatorname{Re}}$

$$
\begin{aligned}
& L^{-1}\left(\frac{1}{s^{1-\gamma}}\right)=h(t)=\frac{1}{t^{\gamma} \Gamma(1-\gamma)} \\
& F_{\gamma}\left(-H_{1}, \tau\right)=L^{-1}\left(\frac{1}{p^{\gamma}+H_{1}}\right) \sum_{n=0}^{\infty} \frac{\left(-H_{i}\right)^{n} \tau^{(n+1) \gamma-1}}{\Gamma((n+1) \gamma)} .
\end{aligned}
$$

$F_{\gamma}(.,$.$) is define in [27] an called as Robotnov and Hartleys' function.$

$$
\chi_{0}=\frac{G\left(1-(-1)^{n}\right)+\sigma_{n}^{2}+\lambda \sigma_{n}^{4}}{\sigma_{n} \operatorname{Re}}
$$

Steady state solution of the given model is given by:

$$
\eta_{p}(\xi)=1-\left(\frac{2-G d^{2}}{2 d}\right) \xi-G-\frac{G \xi^{2}}{2}+G\left\{\frac{\cosh \left(\frac{d-2 \xi}{2}\right)}{\cosh \left(\frac{d}{2}\right)}\right\}
$$

and the transient solution $\eta_{\tau}(\xi, \tau)$ is given as:

$$
\eta_{\tau}(\xi, \tau)=\frac{2}{h} \sum_{n=1}^{\infty} \chi_{0} h(t) * E_{\alpha}\left(-H_{1}, \tau\right) \sin \left(\sigma_{n} \xi\right)
$$




\subsection{Exact Solutions Using Caputo-Fabrizio Definition}

Classical CSF is converted into fractional model by applying Caputo-Fabrizio fractional approach we get the following fractional model of CSF:

$$
{ }^{C F} \mathbf{D}_{\tau}^{\alpha} \operatorname{Re} \eta(\xi, \tau)=G+\eta_{\xi \xi}-\lambda \eta_{\xi \xi \xi \xi},
$$

where ${ }^{C F} \mathbf{D}_{\tau}^{\alpha}$ (, ) CF fractional derivatives which is defined as[17].

$$
{ }^{C F} \mathbf{D}_{\tau}^{\alpha}(\tau)=\frac{1}{1-\alpha} \int_{0}^{\tau} \exp \left(\frac{-\alpha(\tau-t)}{1-\alpha}\right) f^{\prime}(\tau) d t,
$$

Applying both the Laplace and Fourier transforms jointly to Eq. (18) we get:

$$
\bar{\eta}_{s}(n, p)=\frac{\left[G\left(1-(-1)^{n}\right)+\sigma_{n}^{2}+\lambda \sigma_{n}^{4}\right]\left(p+H_{3}\right)}{\sigma_{n} p\left(R_{e} H_{2}+\sigma_{n}^{2}+\lambda \sigma_{n}^{4}\right)\left(p+H_{4}\right)},
$$

After some simplification we get the given equation in more appropriate form as:

$$
\begin{gathered}
\bar{\eta}_{s}(n, p)=\frac{\left[G\left(1-(-1)^{n}\right)+\sigma_{n}^{2}+\lambda \sigma_{n}^{4}\right]}{\sigma_{n}\left(\sigma_{n}^{2}+\lambda \sigma_{n}^{4}\right) p}+\frac{\left[G\left(1-(-1)^{n}\right)+\sigma_{n}^{2}+\lambda \sigma_{n}^{4}\right]}{\sigma_{n}\left(\operatorname{ReH}_{2}+\sigma_{n}^{2}+\lambda \sigma_{n}^{4}\right)\left(p+H_{4}\right)} \\
-\frac{\left[G\left(1-(-1)^{n}\right)+\sigma_{n}^{2}+\lambda \sigma_{n}^{4}\right]}{\sigma_{n}\left(\sigma_{n}^{2}+\lambda \sigma_{n}^{4}\right)\left(p+H_{4}\right)}
\end{gathered}
$$

Applying the Laplace and Fourier inverse transform we get the following solutions:

$$
\begin{gathered}
\eta(\xi, \tau)=1-\left(\frac{2-G d^{2}}{2 d}\right) \xi-G-\frac{G \xi^{2}}{2}+G\left\{\frac{\cosh \left(\frac{d-2 \xi}{2}\right)}{\cosh \left(\frac{d}{2}\right)}\right\}+\frac{2}{d} \sum_{n=1}^{\infty} A_{1} \sin \left(\delta_{n} \xi\right) \exp \left(-H_{4} \tau\right) \\
-\frac{2}{d} \sum_{n=1}^{\infty} A_{2} \sin \left(\delta_{n} \xi\right) \exp \left(-H_{4} \tau\right) .
\end{gathered}
$$

The above solution can be written as:

$$
\eta(\xi, \tau)=1-\left(\frac{2-G d^{2}}{2 d}\right) \xi-G-\frac{G \xi^{2}}{2}+G\left\{\frac{\cosh \left(\frac{d-2 \xi}{2}\right)}{\cosh \left(\frac{d}{2}\right)}\right\}+\frac{2}{d} \sum_{n=1}^{\infty}\left(A_{1}-A_{2}\right) \sin \left(\delta_{n} \xi\right) \exp \left(-H_{4} \tau\right)
$$


From the above solution the steady is given by:

$$
\eta(\xi, \tau)=1-\left(\frac{2-G d^{2}}{2 d}\right) \xi-G-\frac{G \xi^{2}}{2}+G\left\{\frac{\cosh \left(\frac{d-2 \xi}{2}\right)}{\cosh \left(\frac{d}{2}\right)}\right\}
$$

Transient solution is given below:

$$
\eta_{\tau}(\tau)=\frac{2}{d} \sum_{n=1}^{\infty}\left(A_{1}-A_{2}\right) \sin \left(\delta_{n} \xi\right) \exp \left(-H_{4} \tau\right)
$$

where $H_{2}=\frac{1}{1-\alpha}, \delta_{n}=\frac{n \pi}{d}, H_{3}=\frac{\alpha}{1-\alpha}$ and $H_{4}=\frac{\left(\delta_{n}^{2}+\lambda \delta_{n}^{4}\right) H_{3}}{R e H_{2}+\delta_{n}^{2}+\lambda \delta_{n}^{4}}$.

$$
A_{1}=\frac{\left[G\left(1-(-1)^{n}\right)+\delta_{n}^{2}+\lambda \delta_{n}^{4}\right]}{\delta_{n}\left(\operatorname{ReH}_{2}+\delta_{n}^{2}+\lambda \delta_{n}^{4}\right)}, \quad A_{2}=\frac{\left[G\left(1-(-1)^{n}\right)+\delta_{n}^{2}+\lambda \delta_{n}^{4}\right]}{\delta_{n}\left(\delta_{n}^{2}+\lambda \delta_{n}^{4}\right)}
$$

\subsection{Exact Solutions Using Atangana-Baleanu Definition}

The classical couple stress fluid model is transformed fractional model by applying Atangana-Baleanu AB fractional approach we get:

$$
{ }^{A B} \mathbf{D}_{\tau}^{\beta} A \eta(\xi, \tau)=G+\eta_{\xi \xi}-\lambda \eta_{\xi \xi \xi \xi},
$$

$\mathrm{AB}$ time fractional derivatives is defined as [30].

$$
{ }^{A B} \mathbf{D}_{\tau}^{\beta}(\tau)=\frac{N(\beta)}{(1-\beta)} \int_{0}^{\tau} E_{\beta}\left(\frac{-\beta(\tau-t)^{\beta}}{1-\beta}\right) f^{\prime}(\tau) d t
$$

Where $E_{\beta}$ Mittag-Leffler function is used in $\mathrm{AB}$ fractional derivative [31].

$$
E_{\beta}\left(-t^{\beta}\right)=\sum_{k=0}^{\infty} \frac{(-t)^{\beta k}}{\Gamma(\beta k+1)}
$$


Applying the Laplace and Fourier transform we obtain:

$$
\bar{\eta}_{s}(n, p)=\frac{\left[G\left(1-(-1)^{n}\right)+\sigma_{n}^{2}+\lambda \sigma_{n}^{4}\right]}{\sigma_{n}\left(\sigma_{n}^{2}+\lambda \sigma_{n}^{4}\right) p}+\frac{\left[G\left(1-(-1)^{n}\right)+\sigma_{n}^{2}+\lambda \sigma_{n}^{4}\right]\left(M_{2}-M_{3}\right)}{\sigma_{n}\left(R e M_{1}+\sigma_{n}^{2}+\lambda \sigma_{n}^{4}\right)\left(-M_{3}\right)^{\frac{1}{\beta}}\left(p^{\beta}+M_{3}\right)}
$$

Now, applying the inverse Laplace and sine-Fourier transform we obtain the following form [28, 29].

$$
\eta(\xi, \tau)=1-\left(\frac{2-G d^{2}}{2 d}\right) \xi-G-\frac{G \xi^{2}}{2}+G\left\{\frac{\cosh \left(\frac{d-2 \xi}{2}\right)}{\cosh \left(\frac{d}{2}\right)}\right\}+\frac{2}{d} \sum_{n=0}^{\infty} A_{3} \sin \left(\delta_{n} \xi\right) .
$$

Steady solution is given below:

$$
\eta(\xi, \tau)=1-\left(\frac{2-G d^{2}}{2 d}\right) \xi-G-\frac{G \xi^{2}}{2}+G\left\{\frac{\cosh \left(\frac{d-2 \xi}{2}\right)}{\cosh \left(\frac{d}{2}\right)}\right\}
$$

unsteady solutions is given by:

$$
\eta_{\tau}(\tau)=\frac{2}{d} \sum_{n=0}^{\infty} A_{3} \sin \left(\delta_{n} \xi\right)
$$

where

$$
\begin{aligned}
& M_{1}=\frac{1}{1-\beta}, \delta_{n}=\frac{n \pi}{d}, M_{2}=\frac{\beta}{1-\beta} \text { and } M_{3}=\frac{\left(\delta_{n}^{2}+\lambda \delta_{n}^{4}\right) M_{2}}{\operatorname{Re} M_{1}+\delta_{n}^{2}+\lambda \delta_{n}^{4}} . \\
& L^{-1}\left(\frac{1}{s^{\beta}+z}\right)=F_{\beta}(-z, t)=\sum_{n=0}^{\infty} \frac{(-z)^{n} t^{(n+1) \beta-1}}{\Gamma((n+1) \beta)} . \\
& A_{3}=\frac{\left[G\left(1-(-1)^{n}\right)+\delta_{n}^{2}+\lambda \delta_{n}^{4}\right]\left(M_{2}-M_{3}\right)}{\delta_{n}\left(\operatorname{ReM}_{1}+\delta_{n}^{2}+\lambda \delta_{n}^{4}\right)\left(-M_{3}\right)^{\frac{1}{\beta}}} \times \sum_{k=0}^{\infty} \frac{\left(-M_{3}\right)^{k} \tau^{(k+1) \beta-1}}{\Gamma((k+1) \beta)} .
\end{aligned}
$$




\section{Limiting Cases}

\section{A. Newtonian Viscous Fluid}

By putting the CSF parameter $(\lambda=0)$ the following Newtonian solution is obtained:

$$
\begin{aligned}
& \eta(\xi, \tau)=1-G-\left(\frac{1}{d}-\frac{G d}{2}\right) \xi-\frac{G \xi^{2}}{2}+G\left\{\frac{\cosh \left(\frac{d}{2}-\xi\right)}{\cosh \left(\frac{d}{2}\right)}\right\} \\
& +\frac{2}{d} \sum_{n=0}^{\infty}\left(\frac{\left[G\left(1-(-1)^{n}\right)+\delta_{n}^{2}\right]\left(N_{2}-N_{3}\right)}{\delta_{n}\left(\operatorname{ReN}_{1}+\delta_{n}^{2}\right)\left(-N_{3}\right)^{\frac{1}{\beta}}} \times \sum_{k=0}^{\infty} \frac{\left(-N_{3}\right)^{k} \tau^{(k+1) \beta-1}}{\Gamma((k+1) \beta)}\right) \sin \left(\delta_{n} \xi\right),
\end{aligned}
$$

where

$$
N_{1}=\frac{1}{1-\beta}, \delta_{n}=\frac{n \pi}{d}, N_{2}=\frac{\beta}{1-\beta} \text { and } N_{3}=\frac{\left(\delta_{n}^{2}\right) N_{2}}{\operatorname{ReN} N_{1}+\delta_{n}^{2}}
$$

\section{B. Classical CSF Fluid}

By putting $~ \beta \rightarrow 1$ our fractional CSF model reduces to the solutions of Akhtar and Shah[24].

By using the property we have:

By using the property we have:

$$
\begin{aligned}
\lim _{\beta \rightarrow 1}{ }^{A B} \mathbf{D}_{\tau}^{\beta} u(\xi, \tau) & =\lim _{\beta \rightarrow 1} \mathbf{L}^{-1}\left[\mathbf{L}\left\{{ }^{A B} \mathbf{D}_{\tau}^{\beta} u(\xi, \tau)\right\}\right]=\mathbf{L}^{-1}\left\{\lim _{\beta \rightarrow 1} \frac{q^{\beta} \bar{u}(\xi, q)-u(\xi, 0)}{(1-\beta) q^{\beta}+\beta}\right\} \\
= & \mathbf{L}^{-1}\{q \bar{u}(\xi, q)-u(\xi, 0)\}=\mathbf{L}^{-1}\left[\mathbf{L}\left\{u^{\prime}(\xi, \tau)\right\}\right]=u^{\prime}(\xi, \tau) . \\
\eta^{\prime}(\xi, \tau)=1-G & -\left(\frac{1}{d}-\frac{G d}{2}\right) \xi-\frac{G \xi^{2}}{2}+G\left\{\frac{\cosh \left(\frac{d-2 \xi}{2}\right)}{\cosh \left(\frac{d}{2}\right)}\right\}-\frac{2}{d} \sum_{n=1}^{\infty}\left(\frac{G\left(1-(-1)^{n}\right)}{\delta_{n}^{3}\left(1+\delta_{n}^{2}\right)}+\frac{1}{\delta_{n}}\right) \sin \left(\delta_{n} \xi\right) e^{-\left(\frac{\delta_{n}^{2}+\lambda \delta_{n}^{4}}{\operatorname{Re}}\right) \tau}
\end{aligned}
$$

The above solution is reduced to published result which verify our solutions and use as limiting case. 


\section{Special Case}

\subsection{CSF Fluid Without Pressure}

By putting ( $G=0$ ) we get the following solution:

$$
\eta(\xi, \tau)=1-\left(\frac{1}{d}\right) \xi-+\frac{2}{d} \sum_{n=0}^{\infty}\left(\frac{\left[\delta_{n}^{2}+\lambda \delta_{n}^{4}\right]\left(M_{2}-M_{3}\right)}{\delta_{n}\left(\operatorname{Re} M_{1}+\delta_{n}^{2}+\lambda \delta_{n}^{4}\right)\left(-M_{3}\right)^{\frac{1}{\beta}}} \times \sum_{k=0}^{\infty} \frac{\left(-M_{3}\right)^{k} \tau^{(k+1) \beta-1}}{\Gamma((k+1) \beta)}\right) \sin \left(\delta_{n} \xi\right) .
$$

\section{Skin Friction}

The skin friction is given by;

$$
C_{f}(\xi, \tau)=\left(\frac{\partial \eta}{\partial \xi}-\eta \frac{\partial^{3} \eta}{\partial \xi^{3}}\right)
$$

The skin friction for lower plate:

$$
C f_{l p}(0, \tau)=\left(\frac{\partial \eta}{\partial \xi}-\eta \frac{\partial^{3} \eta}{\partial \xi^{3}}\right)
$$

The skin friction for upper plate:

$$
C f_{u p}(1, \tau)=\left(\frac{\partial \eta}{\partial \xi}-\eta \frac{\partial^{3} \eta}{\partial \xi^{3}}\right)
$$

\section{Results And Discussion}

The aim of this section is to analyzed the results through graphs. In the given study we have considered CSF in an open channel bounded by two parallel plates. Figure 1 shows the physical sketch of the problem. The influence of various physical parameters of interest are shown in figures 2 to 10 for clear understanding the behavior of these physical parameters.

We have considered three different time fractional derivatives Caputo, Caputo-Fabrizio and AtanganaBaleanu derivative. The comparison of these fractional operators are highlighted in figure. 2. From the graph it can be seen that CSF velocity shows good memory effect for $\mathrm{AB}$ fractional derivatives as compared to Caputo and $\mathrm{CF}$ fractional derivatives and classical CSF.

It is necessary to discuss here that in the results and discussion section in all the graphs we have compared the classical CSF with time fractional Caputo, $\mathrm{CF}$ and $\mathrm{AB}$ fractional derivatives. Furthermore, in this study we have considered $\gamma, \alpha$ and $\beta$ as fractional operator for Caputo, $\mathrm{CF}$ and $\mathrm{AB}$ derivative respectively.

For the different values of Caputo fractional operator $\gamma$ on CSF velocity is highlighted in figure 3. From the graph the variation in CSF velocity can be noticed. From the figure it is depicted that CSF velocity of fractional order is in between zero and one, while for classical CSF the value is $(\gamma=1)$ are plotted in order to see the 
differences clearly. From the graph it is cleared that for different values of fractional parameter $\gamma$ variation in CSF velocity can be observed. Fractional solution of CSF is the more general solution for the classical solutions when $\gamma=1$ our obtained result is quiet identical to solutions [24] which verify our solutions.

The increasing values of CF fractional operator $\alpha$ on CSF velocity is highlighted in figure 4. From the graph the variation in CSF velocity can be noticed. From the figure it is depicted that CSF velocity of fractional order is in between zero and one, while for classical CSF the value is $(\alpha=1)$ are plotted in order to see the differences clearly. From the graph it is cleared that for different values of fractional parameter $\alpha$ variation in CSF velocity can be observed. Fractional solution of CSF is the more general solution for the classical solutions when $\alpha=1$ our obtained result is quiet identical to solutions [24] which verify our solutions.

The change in CSF velocity for different values of Atangana-Baleanu fractional operator $\beta$ is highlighted in figure 5. From the figure it can be observed that for different values of $\mathrm{AB}$ fractional operator $\beta$ results a variation in CSF velocity. From the figure it is depicted that Couple stress fluid of fractional order and classical CSF are compared in order to observed the differences clearly. It can be noted that for all the three classical CSF cases when $\alpha=\beta=\gamma=1$ our obtained result in all cases reduced to solutions obtained in [24] which verify our solutions.

Figure 6 highlights the comparison of $\mathrm{CSF}$ velocity profile for $\mathrm{C}, \mathrm{CF}, \mathrm{AB}$ and classical velocity with variation of external pressure gradient $G$ when $\operatorname{Re}=0.4, \tau=0.7, \alpha=\beta=\gamma=0.5$ and $\lambda=0.8$. From this figure it is found that increasing the values of $G$ from $G=8$ to $G=10 \mathrm{CSF}$ velocity increases for C,CF, AB and for the case of classical CSF. This behavior of $G$ on CSF velocity is obvious because external pressure accelerates the fluid motion.

Figure 7 shows the variation of Reynolds number $\operatorname{Re}$ on velocity profile when $G=8, \alpha=\beta=\gamma=0.5$, $\tau=0.7$ and $\lambda=0.8$. From this figure one can noticed that for higher Reynolds number $\operatorname{Re}$ from $\operatorname{Re}=0.4$ to $\operatorname{Re}=0.6 \mathrm{CSF}$ velocity decreases. This shows that CSF velocity is controlled by $\mathrm{Re}$ in an open channel.

Figure 8 depicts the comparison of CSF velocity profile for $\mathrm{C}, \mathrm{CF}, \mathrm{AB}$ and classical velocity with variation of time $\tau$ when $G=8, \alpha=\beta=\gamma=0.5, \operatorname{Re}=0.4$ and $\lambda=0.8$. From the graph we see that for the higher values of time from $\tau=0.7$ to $\tau=0.9$ the fractional and classical CSF velocities increase. As we have considered the unsteady CSF in channel which is time dependent. Increasing time CSF velocity is off course increases.

Figure 9 displays the with variation of CSF parameter $\lambda$ when $G=8, \alpha=\beta=\gamma=0.5, \tau=0.7$ and $\operatorname{Re}=0.8$. From the figure CSF velocity is lowered for higher values of $\lambda$. The couple stress parameter is increases from $\lambda=0.8$ to $\lambda=1.2$ as a result fluid velocity decreases. From this graph it is clear that couple stress fluids are more viscous as compare to Newtonian viscous fluid.

The CSF flow with and without $G$ is highlighted in figure 10. From the graphical analysis it is quite clear that CSF velocity with $G$ is higher than CSF velocity without $G$. This figure clearly shows the effect of $G$ on CSF velocity that $G$ is responsible to accelerate the CSF motion in a channel.

The Newtonian viscous fluid is compared with CSF fluid which is highlighted in figure 11. From the graphical results we have CSF velocity with couple tress parameter $\lambda$ this lowered as compared to Newtonian viscous fluid velocity with $\lambda=0$. In other words the velocity of simple Newtonian fluid is higher than CSF velocity. 
Table 1 shows the skin friction variation for lower plate. In this table the numerical values of skin fraction of $\mathrm{C}, \mathrm{CF}, \mathrm{AB}$ and classical results are evaluated and presented in the table 1. Similarly, in table 2 the numerical values of skin friction variation at upper plate are listed. In this table also the numerical values of skin fraction of $\mathrm{C}$, $\mathrm{CF}, \mathrm{AB}$ and classical results are evaluated and presented in the table 2 . In both of the tables the bold numerical values show the skin friction variation for $G, \operatorname{Re}, \tau, \lambda, \alpha, \beta$ and $\gamma$ in that specific row.

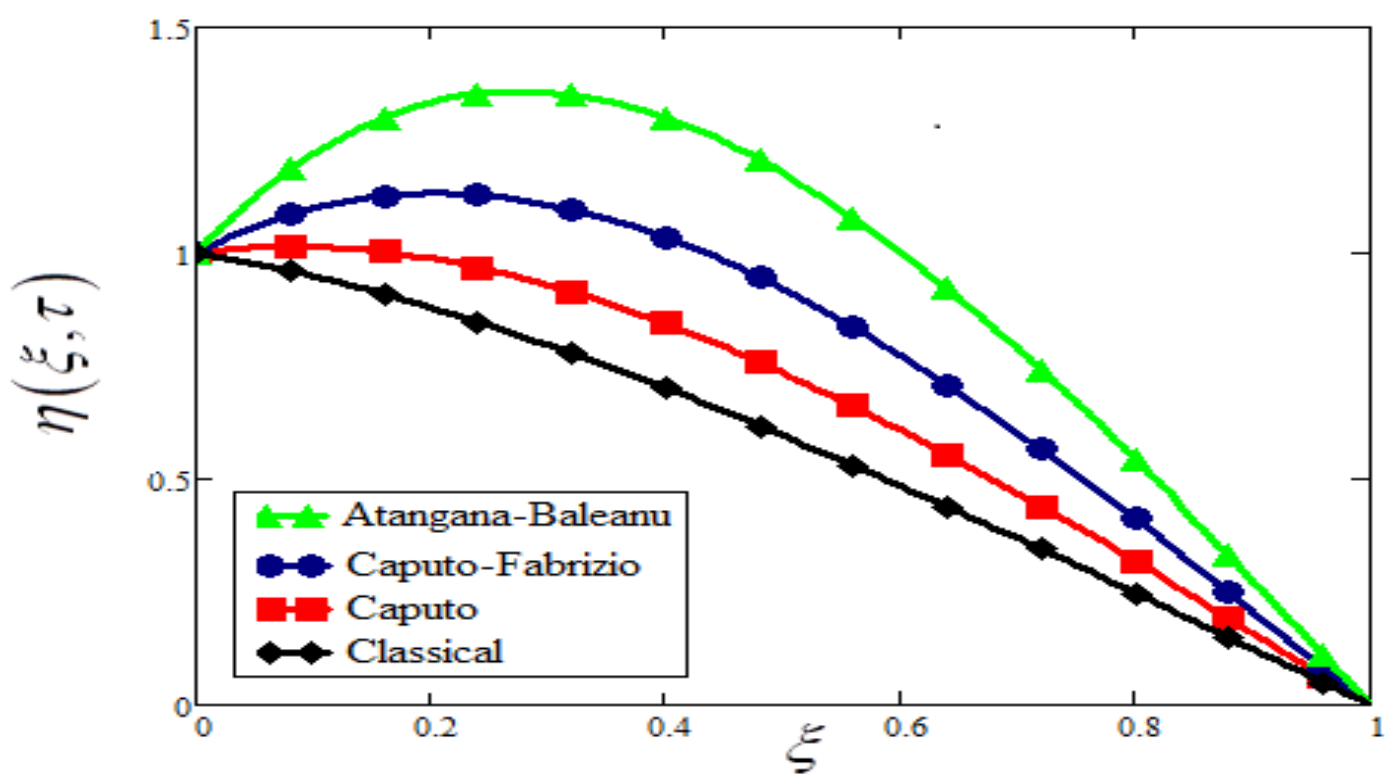

Fig. 2: Comparison of Caputo, $\mathrm{CF}, \mathrm{AB}$ and classical $\mathrm{CSF}$ velocity when $\eta=1.2, G=8, \operatorname{Re}=0.6, t=0.8$,

$$
\alpha=\beta=\gamma=0.5 \text {. }
$$

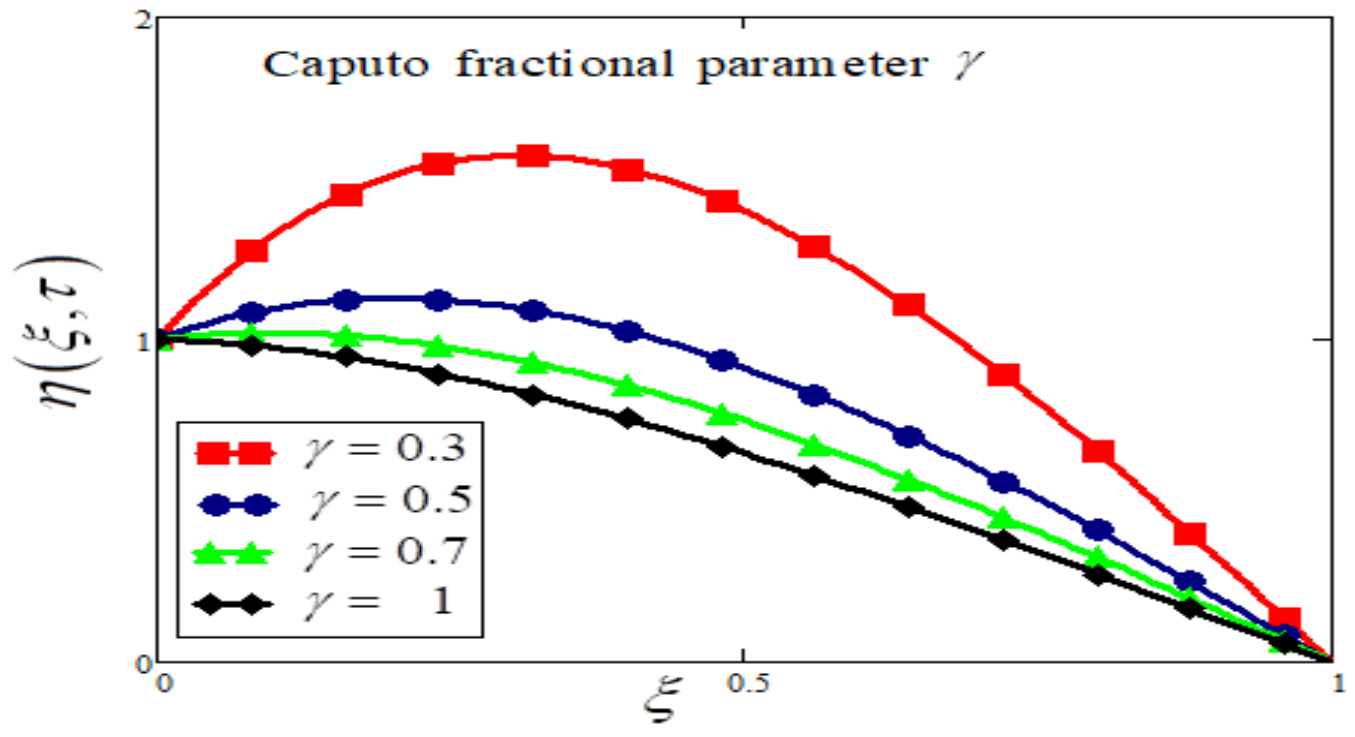

Fig.3: The impact of various values of Caputo fractional parameter $\gamma$ on CSF velocity when $\lambda=1.2, G=10$,

$$
\operatorname{Re}=0.6, t=0.8
$$




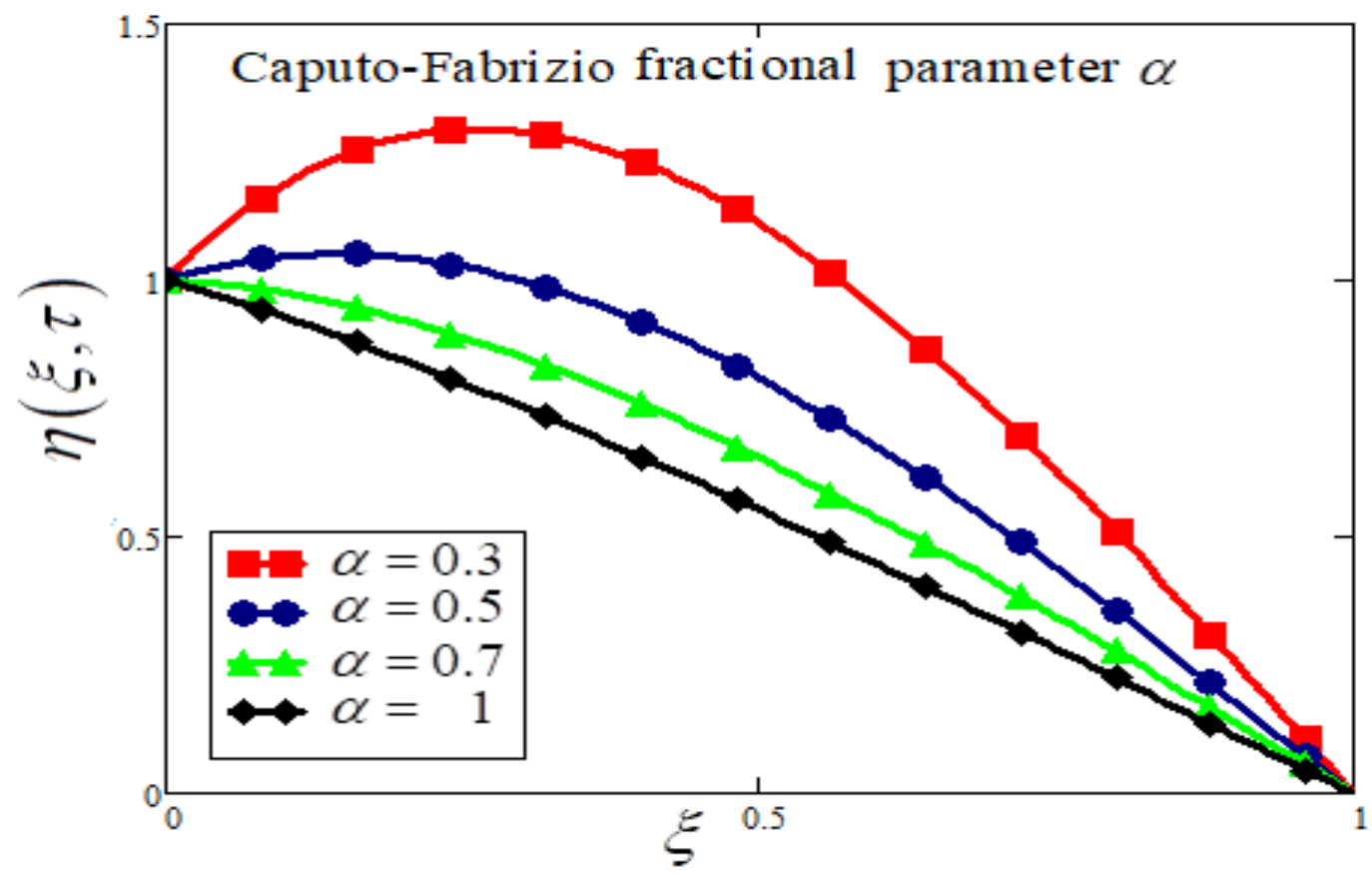

Fig. 4: The impact of various values of CF fractional parameter $\alpha$ on CSF velocity when $\lambda=1.2, G=10$, $\operatorname{Re}=0.6, t=0.6$.

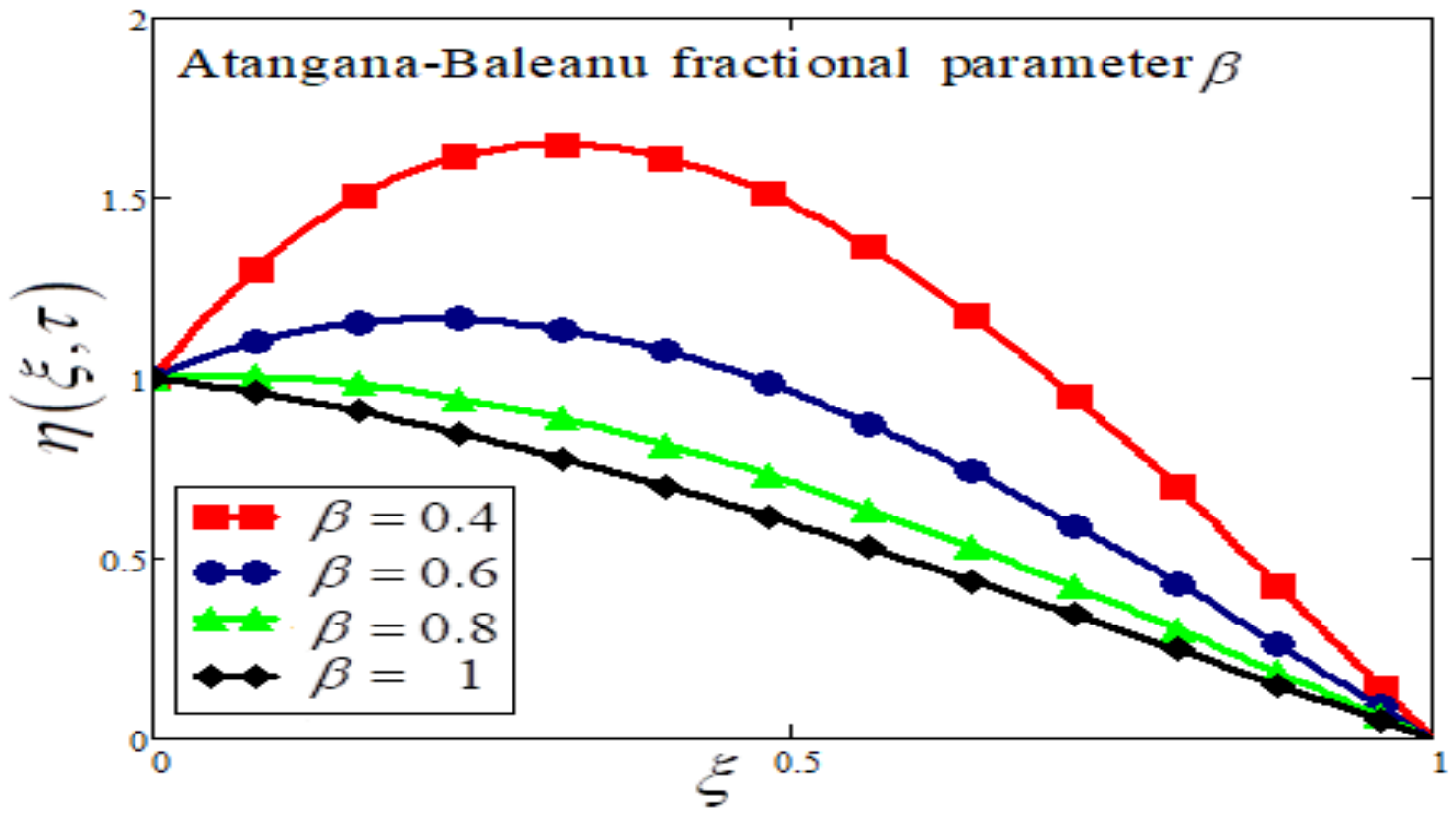

Fig. 5: The impact of $\mathrm{AB}$ fractional operator on $\mathrm{CSF}$ velocity when $\lambda=1.2, G=8, \operatorname{Re}=0.6, \tau=0.8$. 

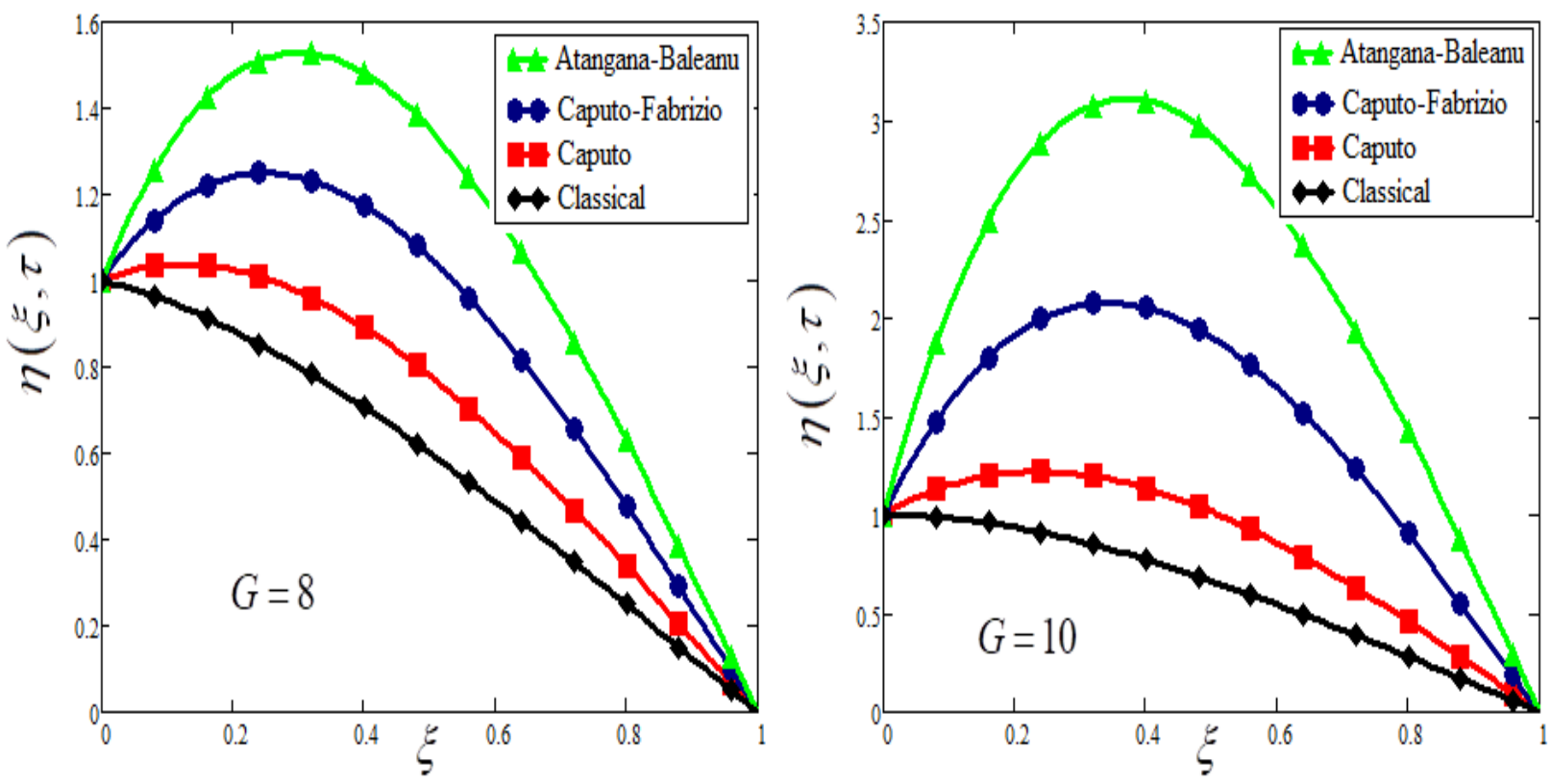

Fig. 6: The impact of external pressure gradient $G$ on $\mathrm{CSF}$ velocity for $\mathrm{C}, \mathrm{CF}, \mathrm{AB}$ and classical velocity when $\operatorname{Re}=0.4, \tau=0.7, \alpha=\beta=\gamma=0.5$ and $\lambda=0.8$.
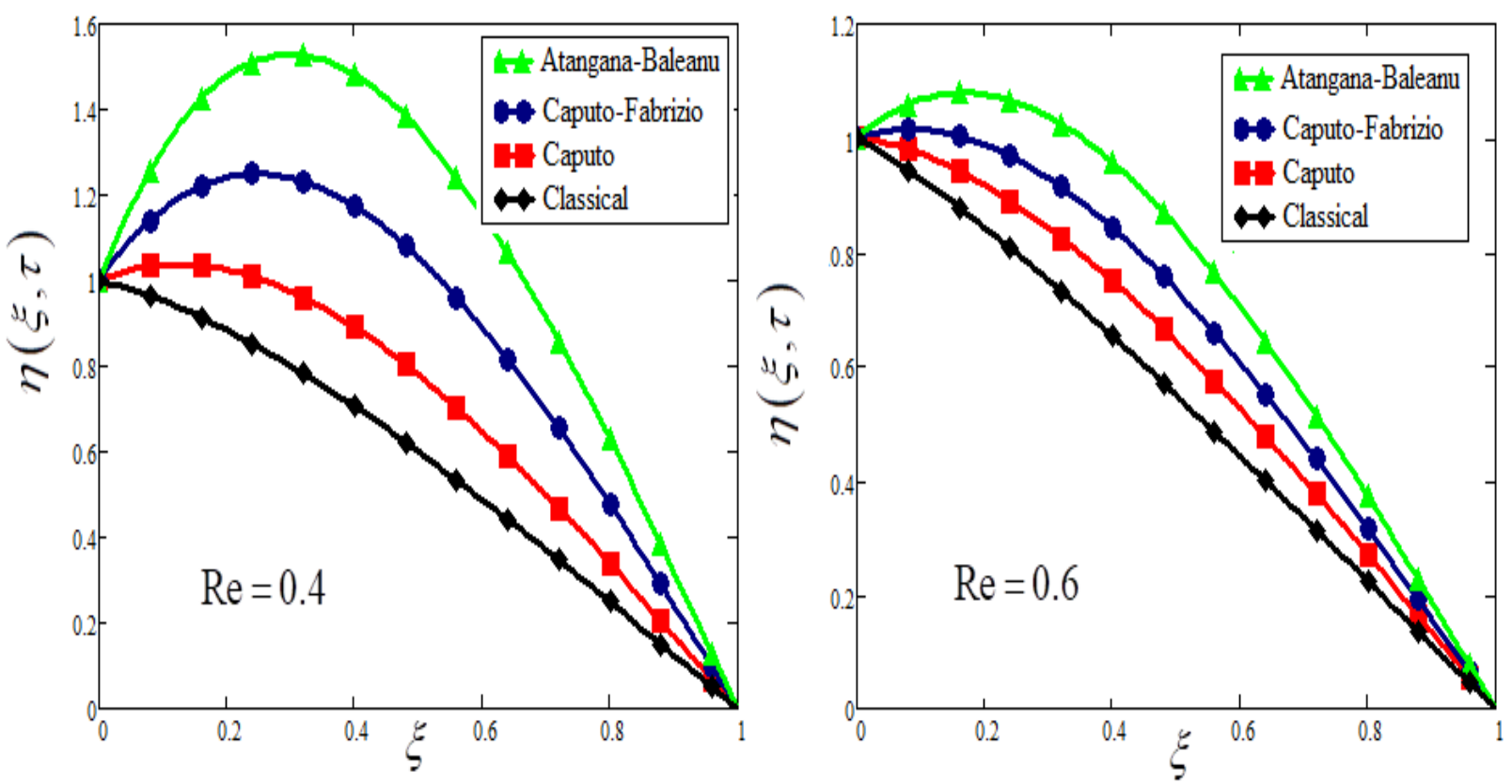

Fig. 7: The impact of Reynolds number $\mathrm{Re}$ on $\mathrm{CSF}$ velocity for $\mathrm{C}, \mathrm{CF}, \mathrm{AB}$ and classical velocity when $G=8$, $\alpha=\beta=\gamma=0.5, \tau=0.7$ and $\lambda=0.8$. 

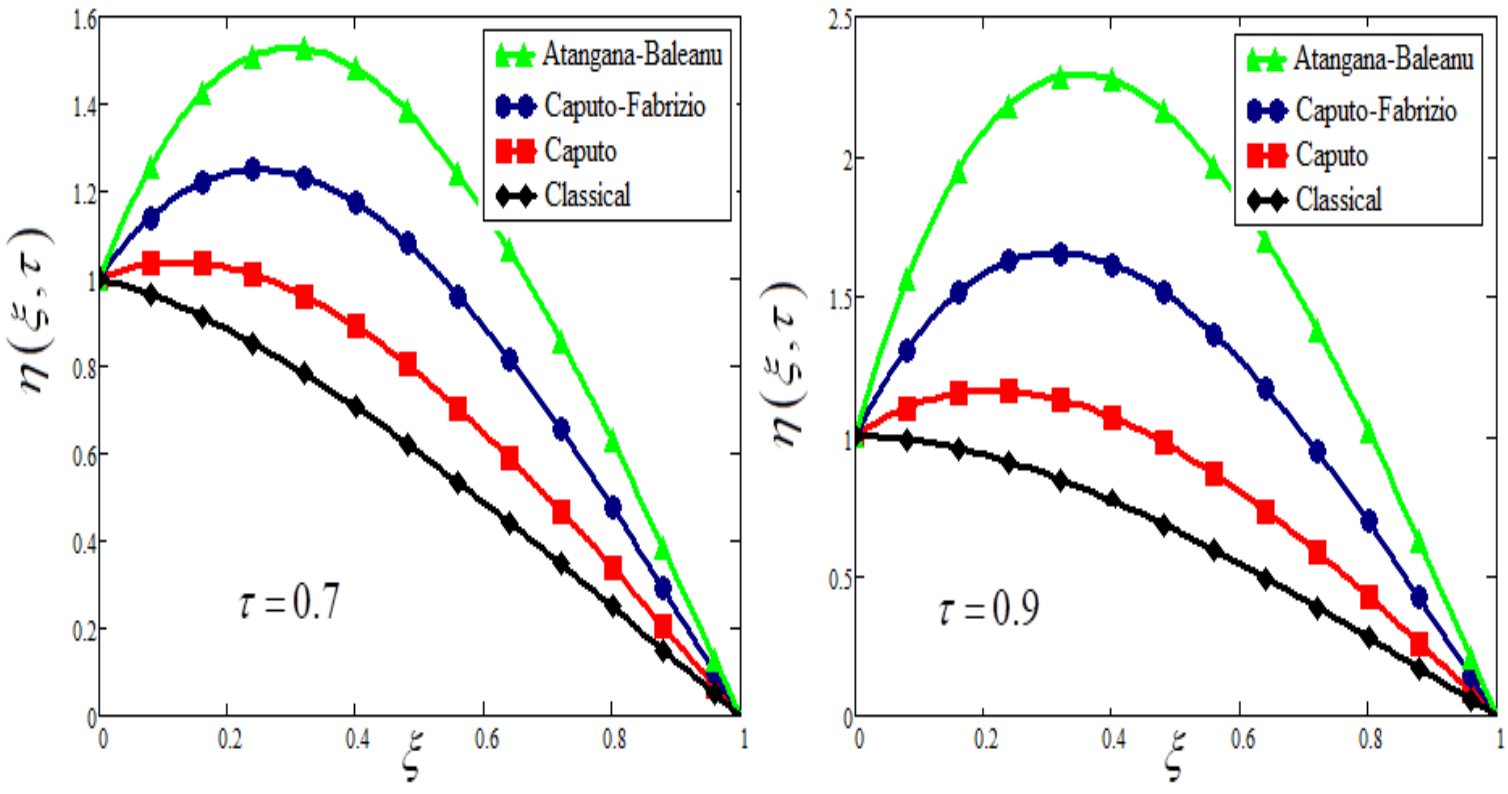

Fig. 8: The impact of time $\tau$ on velocity profile for $\mathrm{C}, \mathrm{CF}, \mathrm{AB}$ and classical $\mathrm{CSF}$ when $G=8, \alpha=\beta=\gamma=0.5$ , $\operatorname{Re}=0.4$ and $\lambda=0.8$.
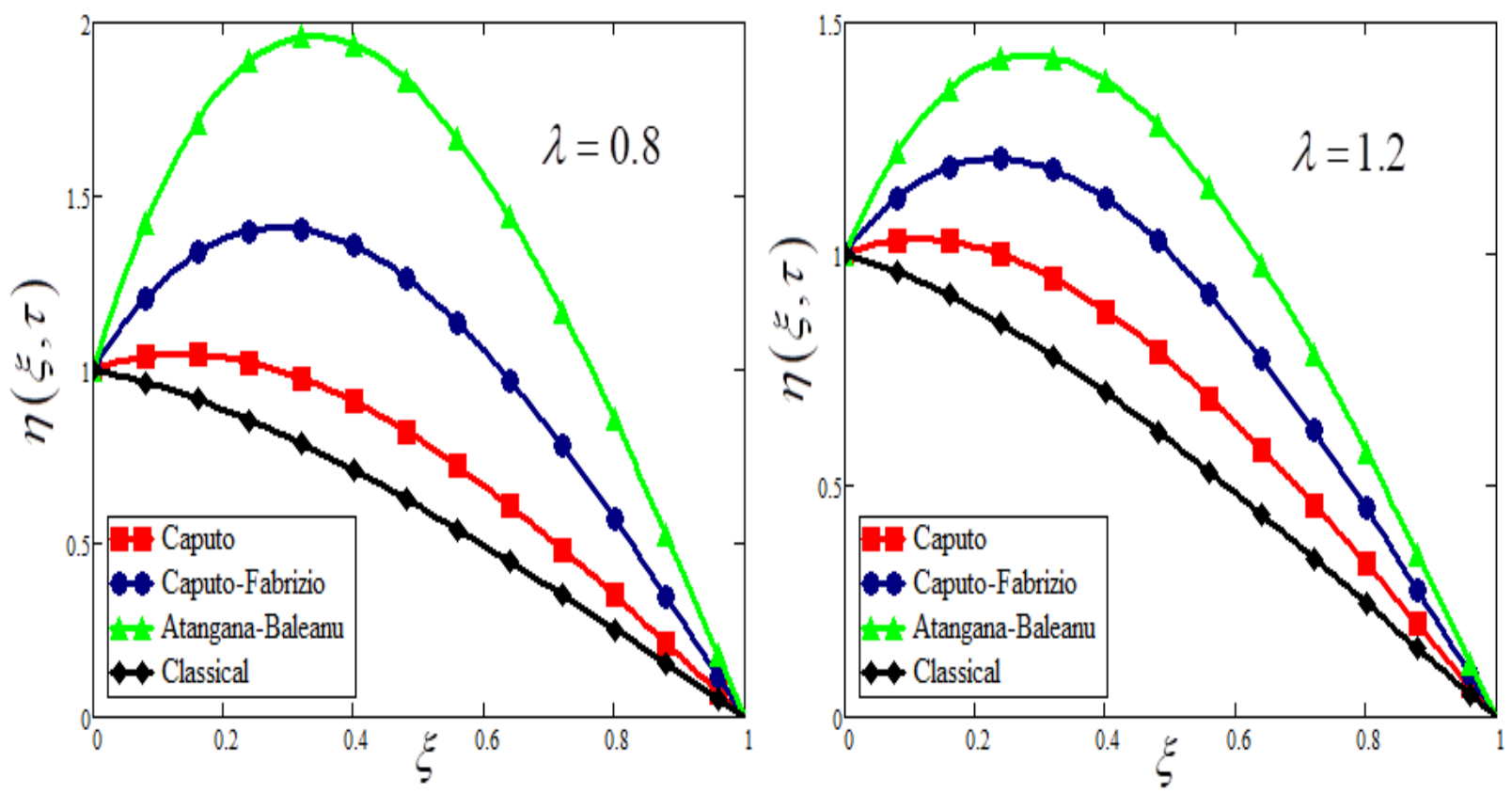

Fig. 9: Comparison of $\mathrm{CSF}$ velocity profile for $\mathrm{C}, \mathrm{CF}, \mathrm{AB}$ and classical velocity with variation of CSF parameter $\lambda$ when $G=8, \alpha=\beta=\gamma=0.5, \tau=0.7$ and $\operatorname{Re}=0.8$. 


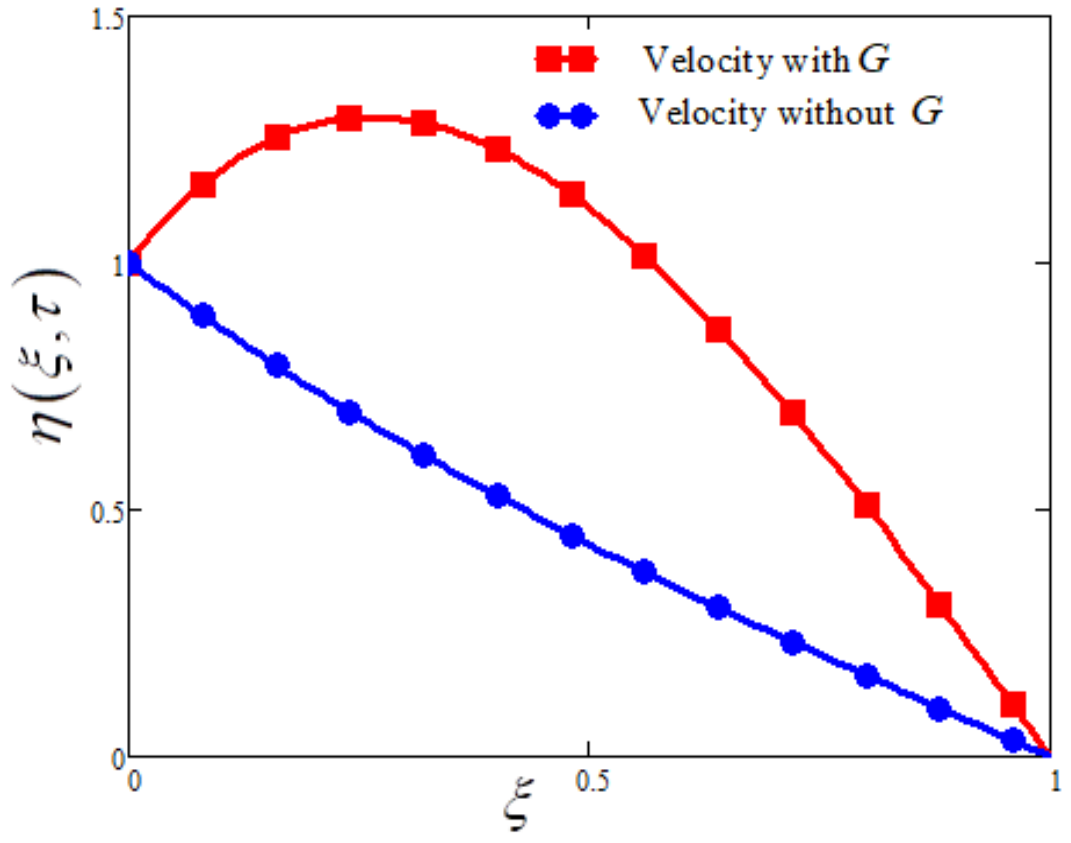

Fig.10: $\mathrm{CSF}$ velocity with $\mathrm{G}$ and without $\mathrm{G}$ when $\mathrm{Re}=0.6, \tau=0.6, \alpha=0.5$ and $\lambda=1.2$

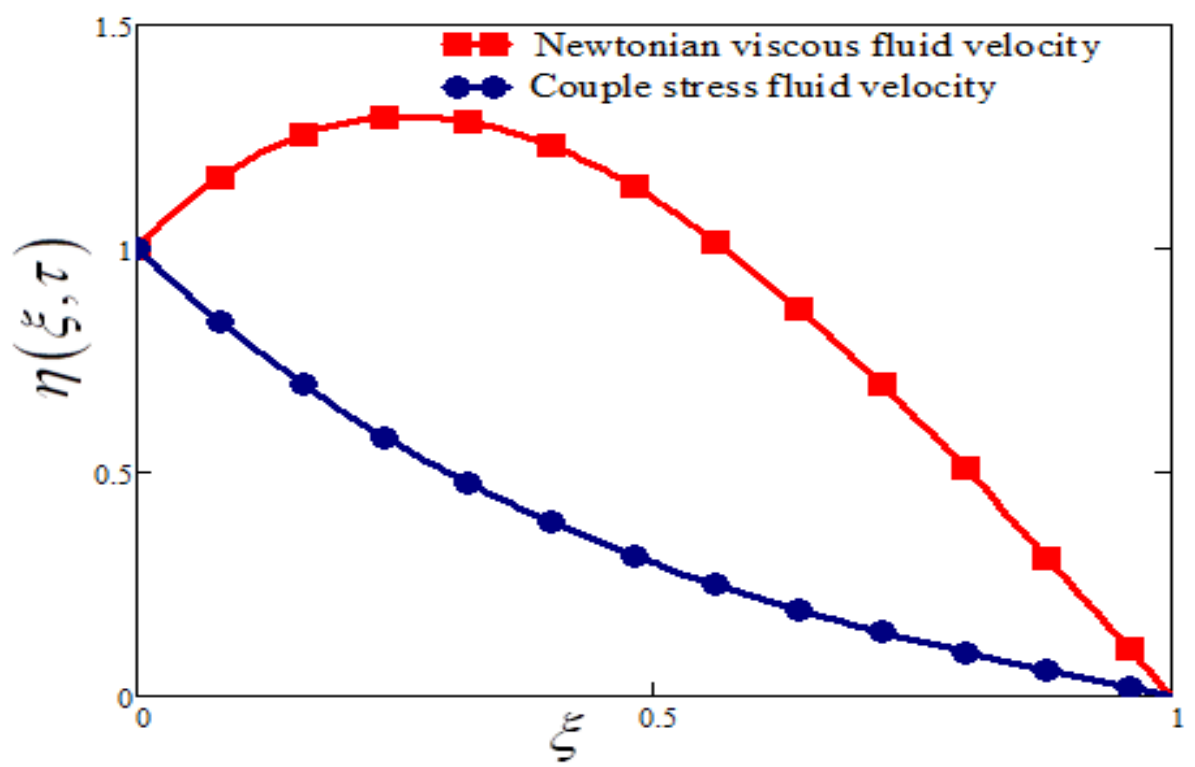

Fig.11: CSF and Newtonian fluid velocity when $G=8, \operatorname{Re}=0.6$ and $\tau=0.7$. 
Table1. The skin friction at lower plate for Caputo, CF, AB and classical CSF.

\begin{tabular}{lllllllllll}
\hline$G$ & $\operatorname{Re}$ & $\tau$ & $\lambda$ & $\gamma$ & $\alpha$ & $\beta$ & $C f_{\gamma}$ & $C f_{\alpha}$ & $C f_{\beta}$ & $C f_{\text {classic }}$ \\
\hline 8 & 0.6 & 0.8 & 1.2 & 0.5 & 0.5 & 0.5 & 0.356 & 1.372 & 2.79 & 0.41 \\
$\mathbf{9}$ & 0.6 & 0.8 & 1.2 & 0.5 & 0.5 & 0.5 & 0.805 & 2.586 & 5.103 & 0.259 \\
8 & $\mathbf{0 . 8}$ & 0.8 & 1.2 & 0.5 & 0.5 & 0.5 & 0.144 & 0.261 & 0.887 & 0.603 \\
8 & 0.6 & $\mathbf{0 . 9}$ & 1.2 & 0.5 & 0.5 & 0.5 & 0.996 & 2.751 & 5.413 & 0.148 \\
8 & 0.6 & 0.8 & $\mathbf{1 . 4}$ & 0.5 & 0.5 & 0.5 & 0.33 & 1.39 & 2.902 & 0.442 \\
8 & 0.6 & 0.8 & 1.2 & $\mathbf{0 . 7}$ & 0.5 & 0.5 & 0.09 & 1.372 & 2.79 & 0.41 \\
8 & 0.6 & 0.8 & 1.2 & 0.5 & $\mathbf{0 . 7}$ & 0.5 & 0.356 & 0.269 & 2.79 & 0.41 \\
8 & 0.6 & 0.8 & 1.2 & 0.5 & 0.5 & $\mathbf{0 . 7}$ & 0.356 & 1.372 & 0.767 & 0.41 \\
\hline
\end{tabular}

Table2. The skin friction at upper plate for Caputo, CF, AB and classical CSF.

\begin{tabular}{ccccccccccc}
\hline$G$ & $\operatorname{Re}$ & $\tau$ & $\lambda$ & $\gamma$ & $\alpha$ & $\beta$ & $C f_{\gamma}$ & $C f_{\alpha}$ & $C f_{\beta}$ & $C f_{\text {classic }}$ \\
\hline 8 & 0.6 & 0.8 & 1.2 & 0.5 & 0.5 & 0.5 & 1.611 & 2.1 & 2.778 & 1.244 \\
$\mathbf{9}$ & 0.6 & 0.8 & 1.2 & 0.5 & 0.5 & 0.5 & 1.821 & 2.675 & 3.879 & 1.312 \\
8 & $\mathbf{0 . 7}$ & 0.8 & 1.2 & 0.5 & 0.5 & 0.5 & 1.471 & 1.767 & 2.193 & 1.192 \\
8 & 0.6 & $\mathbf{0 . 9}$ & 1.2 & 0.5 & 0.5 & 0.5 & 1.912 & 2.754 & 4.027 & 1.364 \\
8 & 0.6 & 0.8 & $\mathbf{1 . 3}$ & 0.5 & 0.5 & 0.5 & 1.61 & 2.112 & 2.817 & 1.245 \\
8 & 0.6 & 0.8 & 1.2 & $\mathbf{0 . 7}$ & 0.5 & 0.5 & 1.396 & 2.1 & 2.778 & 1.244 \\
8 & 0.6 & 0.8 & 1.2 & 0.5 & $\mathbf{0 . 7}$ & 0.5 & 1.611 & 1.571 & 2.778 & 1.244 \\
8 & 0.6 & 0.8 & 1.2 & 0.5 & 0.5 & $\mathbf{0 . 7}$ & 1.611 & 2.1 & 1.81 & 1.244 \\
\hline
\end{tabular}

\section{Concluding Remarks}

The purpose of the present work is to evaluate and obtained the closed form solutions for the couple stress fluid in an open channel using the integral transforms (Laplace and Fourier). The results obtained for Caputo, CF, $\mathrm{AB}$ and classical CSF are compared through graphs. Furthermore, the effect of some physical parameters are depicted which effect the CSF motion. Finally, some special cases were reproduced to published results which verify our obtained solutions. Additionally, from the comparative analysis of $\mathrm{C}, \mathrm{CF}, \mathrm{AB}$ and classical CSF through graphs we can observed that $\mathrm{CSF}$ velocity for $\mathrm{AB}$ fractional derivative shows good memory effect of fluid dynamics as compared to $\mathrm{C}$ and $\mathrm{CF}$ and the classical CSF velocity.

Some important points are noted during this study which are given below. 
- The influence of $\mathrm{C}, \mathrm{CF}, \mathrm{AB}$ fractional derivatives and classical CSF is displayed in the velocity profile. From the figure it can be seen that CSF velocity for $\mathrm{AB}$ fractional derivative is maximum as compared to all other velocities. It is also noted that classical CSF velocity have minimum magnitude.

- Increasing $G$ CSF velocity increases.

- Increasing Re the magnitude of CSF velocity decreases in cases of C,CF,AB and classical CSF.

- Increasing $\tau$ velocity of the CSF increase.

- $\mathrm{AB}$ fractional derivatives have good memory effect as compared to $\mathrm{C}, \mathrm{CF}$ and Classical CSF.

\section{Declaration of Competing Interest}

The authors declare that they have no known competing financial interest or personal relationships that could have appeared to influence the work reported in this paper.

\section{Acknowledgments:}

The authors acknowledge the financial support provided by the Center of Excellence in Theoretical and Computational Science (TaCS-CoE), KMUTT.

Moreover, this research project is supported by Thailand Science Research and Innovation (TSRI) Basic Research Fund: Fiscal year 2021 under project number 64A306000005.

\section{References}

[1]. Stokes, V. K. Couple stresses in fluids. The physics of fluids, 9(9), 1709-1715. https://doi.org/10.1007/978-3-642-82351-0 4, (1966).

[2]. Sha, Z., Dawar, A., Alzahrani, E. O., Kumam, P., Khan, A. J., \& Islam, S. Hall effect on couple stress 3D nanofluid flow over an exponentially stretched surface with Cattaneo Christov heat flux model. IEEE Access, 7, 64844-64855. https://doi.org/10.1109/ACCESS.2019.2916162. (2019).

[3]. Nagaraju, G., Srinivas, J., Murthy, J. R., \& Rashad, A. M. Entropy generation analysis of the MHD flow of couple stress fluid between two concentric rotating cylinders with porous lining. Heat Transfer-Asian Research, 46(4), 316-330. https://doi.org/10.1002/htj.21214. (2017).

[4]. Kumar, M., Reddy, G. J., Kumar, N. N., \& Bég, O. A. Computational study of unsteady couple stress magnetic nanofluid flow from a stretching sheet with Ohmic dissipation. Proceedings of the Institution of Mechanical Engineers, Part N: Journal of Nanomaterials, Nanoengineering and Nanosystems, 233(2-4), 49-63. https://doi.org/10.1177/2397791419843730 (2019).

[5]. Khan, N. A., Khan, N. A., Ullah, S., \& Naz, F. Swirling flow of couple stress fluid due to a rotating disk. Nonlinear Engineering, 8(1), 261-269. https://doi.org/10.1515/nleng-2017-0062.(2019).

[6]. Hayat, T., Ayub, T., Muhammad, T., \& Ahmad, B. Nonlinear computational treatment for couple stress fluid flow with Cattaneo-Christov double diffusion and homogeneous-heterogeneous reactions. International Journal of Chemical Reactor Engineering, 17(1). https://doi.org/10.1515/ijcre2018-0056. (2018).

[7]. Ahmed, S., Bég, O. A., \& Ghosh, S. K. A couple stress fluid modeling on free convection oscillatory hydromagnetic flow in an inclined rotating channel. Ain Shams Engineering Journal, 5(4), 1249-1265. https://doi.org/10.1016/j.asej.2014.04.006. (2014).

[8]. Bég, O. A., Ghosh, S. K., Ahmed, S., \& Beg, T. Mathematical modeling of oscillatory magneto-convection of a couple-stress biofluid in an inclined rotating channel. Journal of Mechanics in Medicine and Biology, 12(03), 1250050. https://doi.org/10.1142/S0219519411004654. (2012).

[9]. Yadav, D., Mahabaleshwar, U. S., Wakif, A., \& Chand, R. Significance of the inconstant viscosity and internal heat generation on the occurrence of Darcy-Brinkman convective motion in a couple-stress fluid 
saturated porous medium: An analytical solution. International Communications in Heat and Mass Transfer, 122, 105165. https://doi.org/10.1016/j.icheatmasstransfer.2021.105165.(2021).

[10]. Devakar, M., Sreenivasu, D., \& Shankar, B. Analytical solutions of some fully developed flows of couple stress fluid between concentric cylinders with slip boundary conditions. International Journal of Engineering Mathematics, 785396. https://doi.org/10.1155/2014/785396. (2014).

[11]. Naeem, K. A class of flows for couple stress fluids. J. Basic Appl. Sci., 97-104. (2012).

[12]. Mekheimer, K. S. Peristaltic flow of a couple stress fluid in an annulus: application of an endoscope. Physica A: Statistical Mechanics and its Applications, 387(11), 2403-2415. https://doi.org/10.1016/i.physa.2007.12.017.(2008).

[13]. Oldham, K., \& Spanier, J. The fractional calculus theory and applications of differentiation and integration to arbitrary order (Vol. 111). Elsevier. (1974).

[14]. Marks, R. J. I. I., \& Hall, M. Differintegral interpolation from a bandlimited signal's samples. IEEE Transactions on Acoustics, Speech, and Signal Processing, 29(4), 872-877. https://doi.org/10.1109/TASSP.1981.1163636.(1981).

[15]. Olmstead, W. E., \& Handelsman, R. A. Diffusion in a semi-infinite region with nonlinear surface dissipation. SIAM Review, 18(2), 275-291. https://doi.org/10.1137/1018044. (1976).

[16]. Benson, D. A. The fractional advection-dispersion equation: Development and application (Doctoral dissertation, University of Nevada, Reno). (1998).

[17]. Podlubny, I. Fractional differential equations: an introduction to fractional derivatives, fractional differential equations, to methods of their solution and some of their applications. Elsevier. (1998).

[18]. Caputo, M., \& Fabrizio, M. A new definition of fractional derivative without singular kernel. Progr. Fract. Differ. Appl, 1(2), 1-13. (2015).

[19]. Atangana, A., \& Baleanu, D. New fractional derivatives with nonlocal and non-singular kernel: theory and application to heat transfer model. arXiv preprint arXiv:1602.03408. (2016).

[20]. Arif, M., Ali, F., Sheikh, N. A., Khan, I., \& Nisar, K. S. Fractional Model of Couple Stress Fluid for Generalized Couette Flow: A Comparative Analysis of Atangana-Baleanu and Caputo-Fabrizio Fractional Derivatives. IEEE Access, 7, 88643-88655. https://doi.org/10.1109/ACCESS.2019.2925699. (2019).

[21]. Akhtar, S. Flows between two parallel plates of couple stress fluids with time-fractional Caputo and Caputo-Fabrizio derivatives. The European Physical Journal Plus, 131(11), 401. https://doi.org/10.1140/epjp/i2016-16401-3 (2016).

[22]. Hadjesfandiari, A. R., Hajesfandiari, A., \& Dargush, G. F. Skew-symmetric couple-stress fluid mechanics. Acta Mechanica, 226(3), 871-895. https://doi.org/10.1007/s00707-014-1223-0 (2015).

[23]. Farooq, M., Rahim, M. T., Islam, S., \& Siddiqui, A. M. Steady Poiseuille flow and heat transfer of couple stress fluids between two parallel inclined plates with variable viscosity. Journal of the Association of Arab $\begin{array}{lllll}\text { Universities for Basic and } & \text { Applied }\end{array}$ https://doi.org/10.1016/j.jaubas.2013.01.004 .(2013).

[24]. Akhtar, S., \& Shah, N. A. Exact solution s for some unsteady flows of a couple stress fluid between parallel plates. Ain Shams Engineering Journal. https://doi.org/10.1016/j.asej.2016.05.008 .(2016).

[25]. Miller, K. S., \& Ross, B. An introduction to the fractional calculus and fractional differential equations. Wiley. (1993).

[26]. Kilbas, A. A. A., Srivastava, H. M., \& Trujillo, J. J. Theory and applications of fractional differential equations (Vol. 204). Elsevier Science Limited. (2006).

[27]. Lorenzo, C. F., \& Hartley, T. T. Generalized functions for the fractional calculus. (1999).

[28]. Debnath L, Bhatta D. Integral transforms and their applications. 2nd ed. Chapman and Hall/C.R.C.; 2006.

[29]. Gradshteyn IS, Ryzhik IM. Table of integrals, series, and products. 7th ed. Elsevier Academic Press

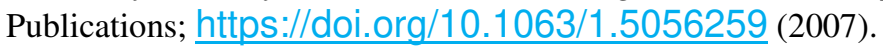

[30]. Atangana A. On the new fractional derivative and application to nonlinear Fisher's reaction-diffusion equation. Appl Math Comput;273:948-56. https://doi.org/10.1016/j.amc.2015.10.021 . (2016).

[31]. Kilbas, A. A., Saigo, M., \& Saxena, R. K. Generalized Mittag-Leffler function and generalized fractional calculus operators. Integral Transforms and Special Functions, 15(1), 31-49. https://doi.org/10.1080/10652460310001600717.(2004). 
Figures

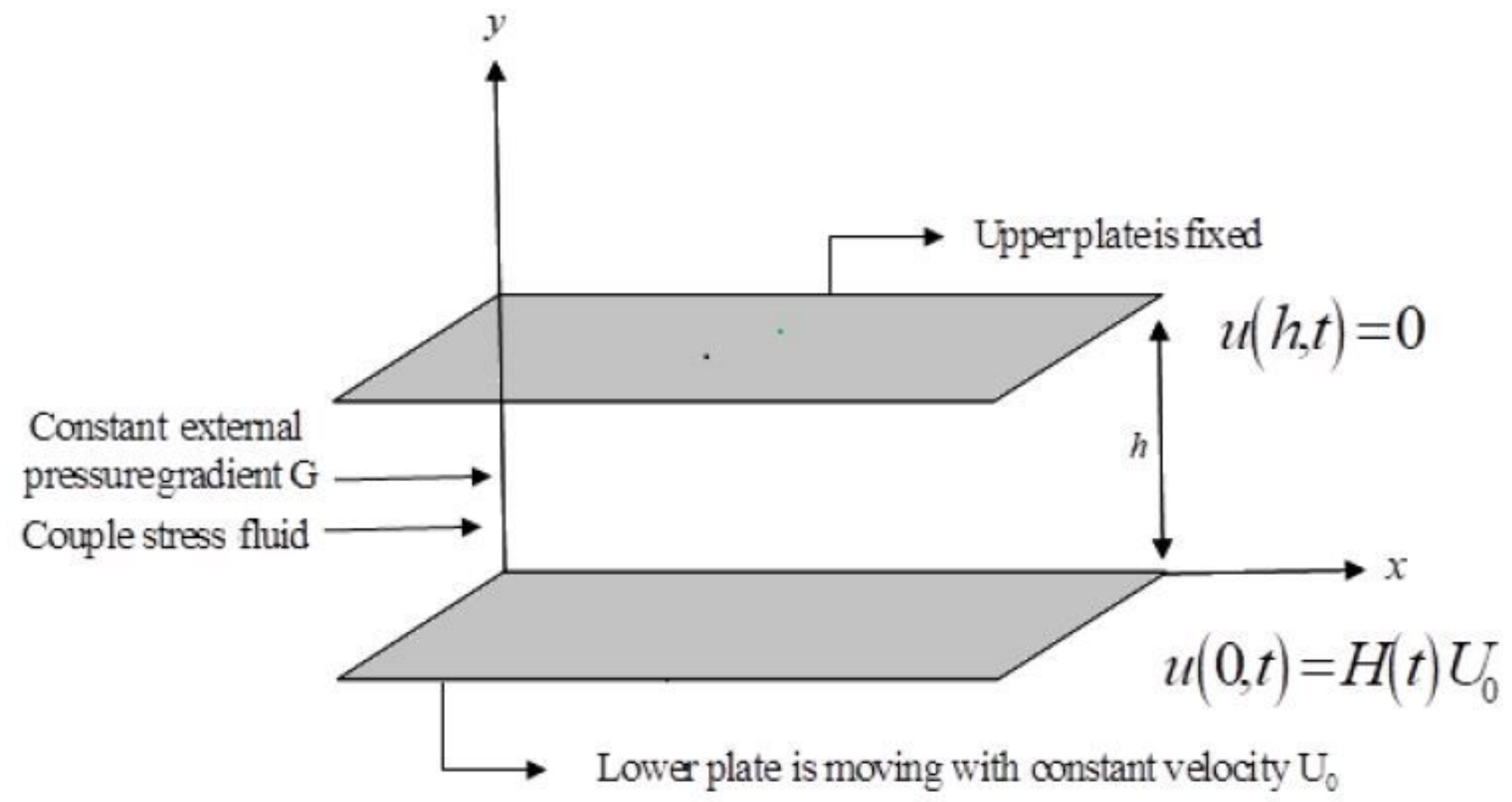

Figure 1

Physical sketch of the problem

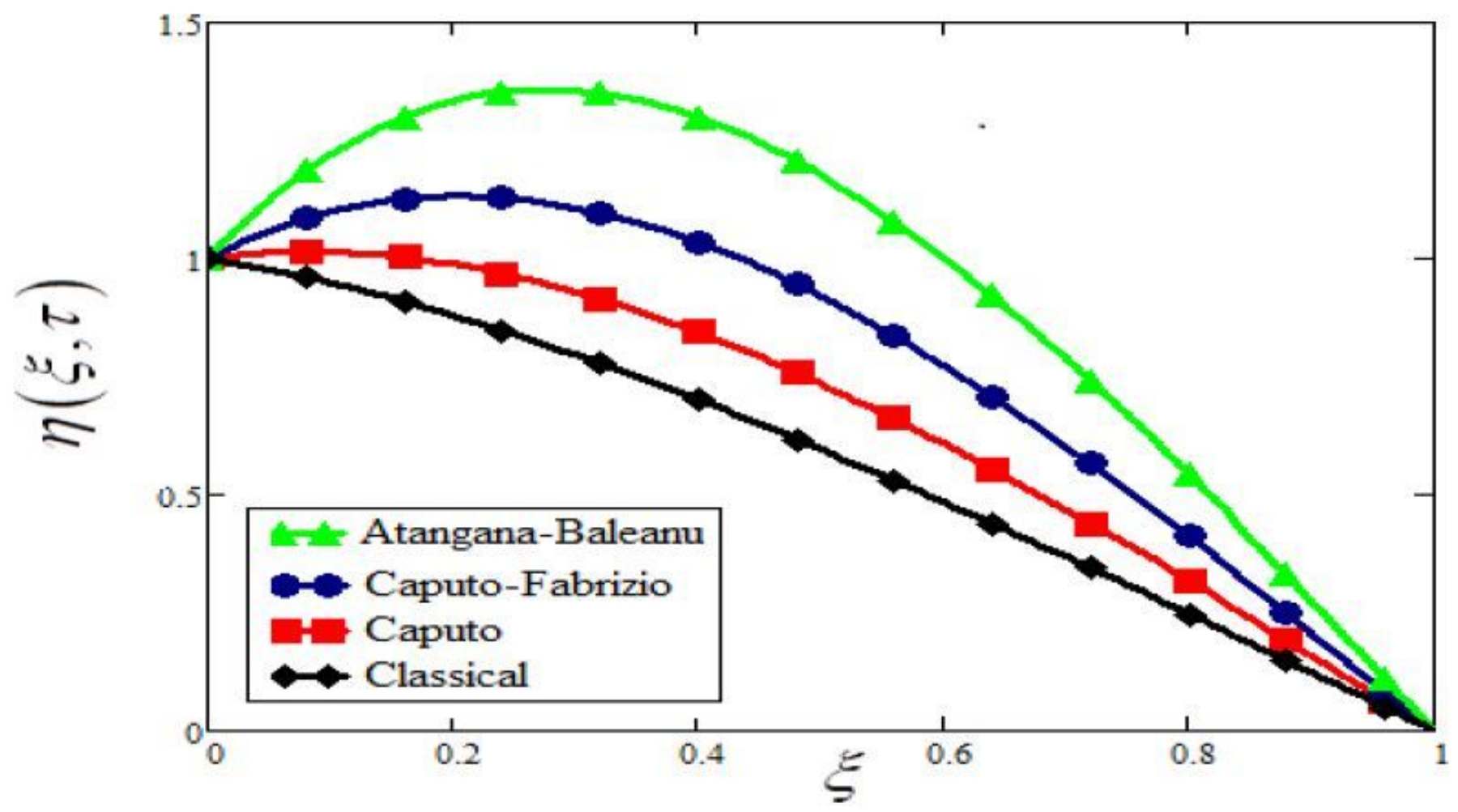


Figure 2

Comparison of Caputo, CF, AB and classical CSF velocity when $\nabla=1.2, G=8, \operatorname{Re}=0.6, t=0.8, a=\beta=\gamma=0.5$.

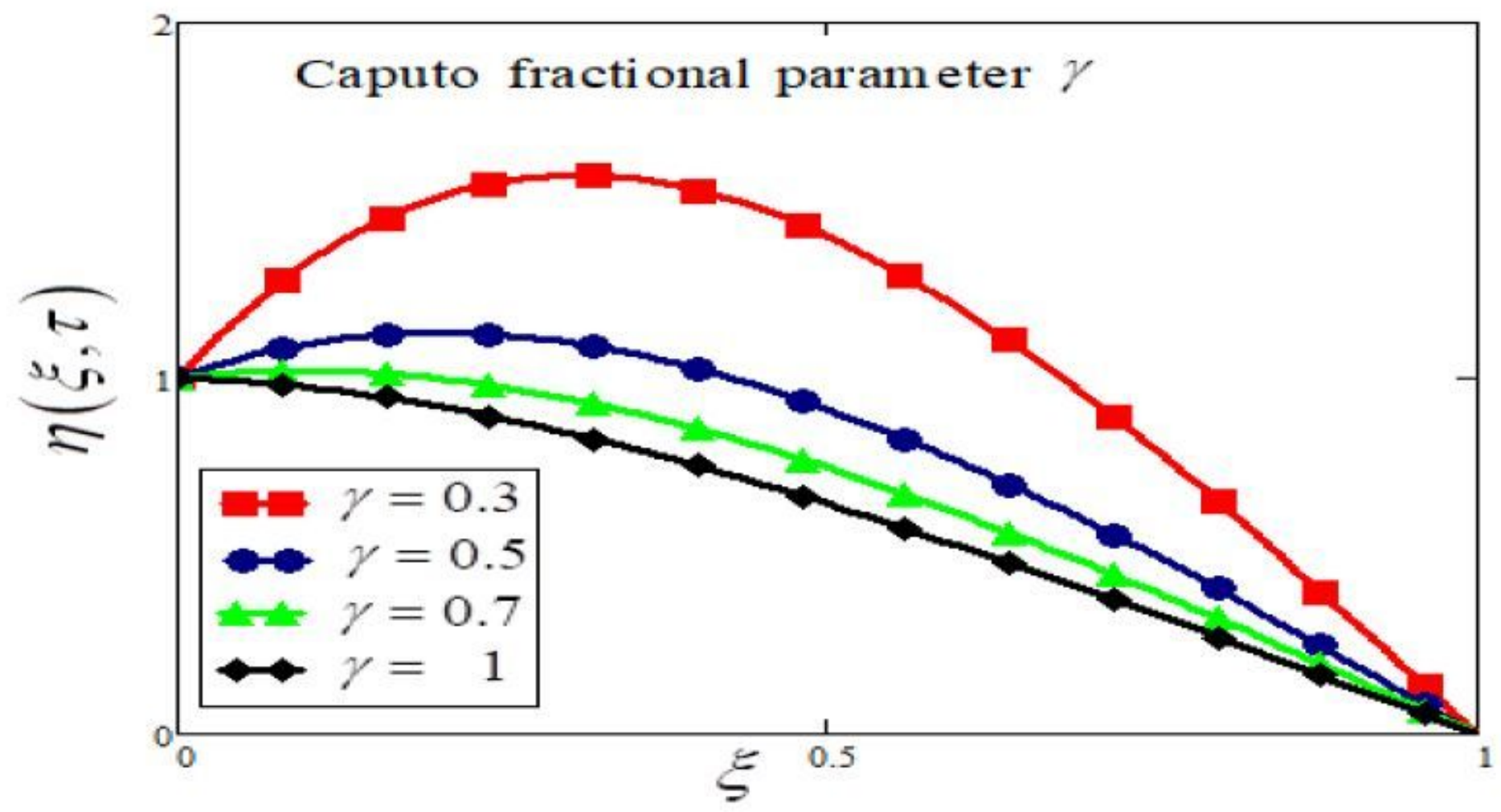

Figure 3

The impact of various values of Caputo fractional parameter $y$ on CSF velocity when $\lambda=1.2, G=10, \operatorname{Re}=0.6$, $\mathrm{t}=0.8$. 


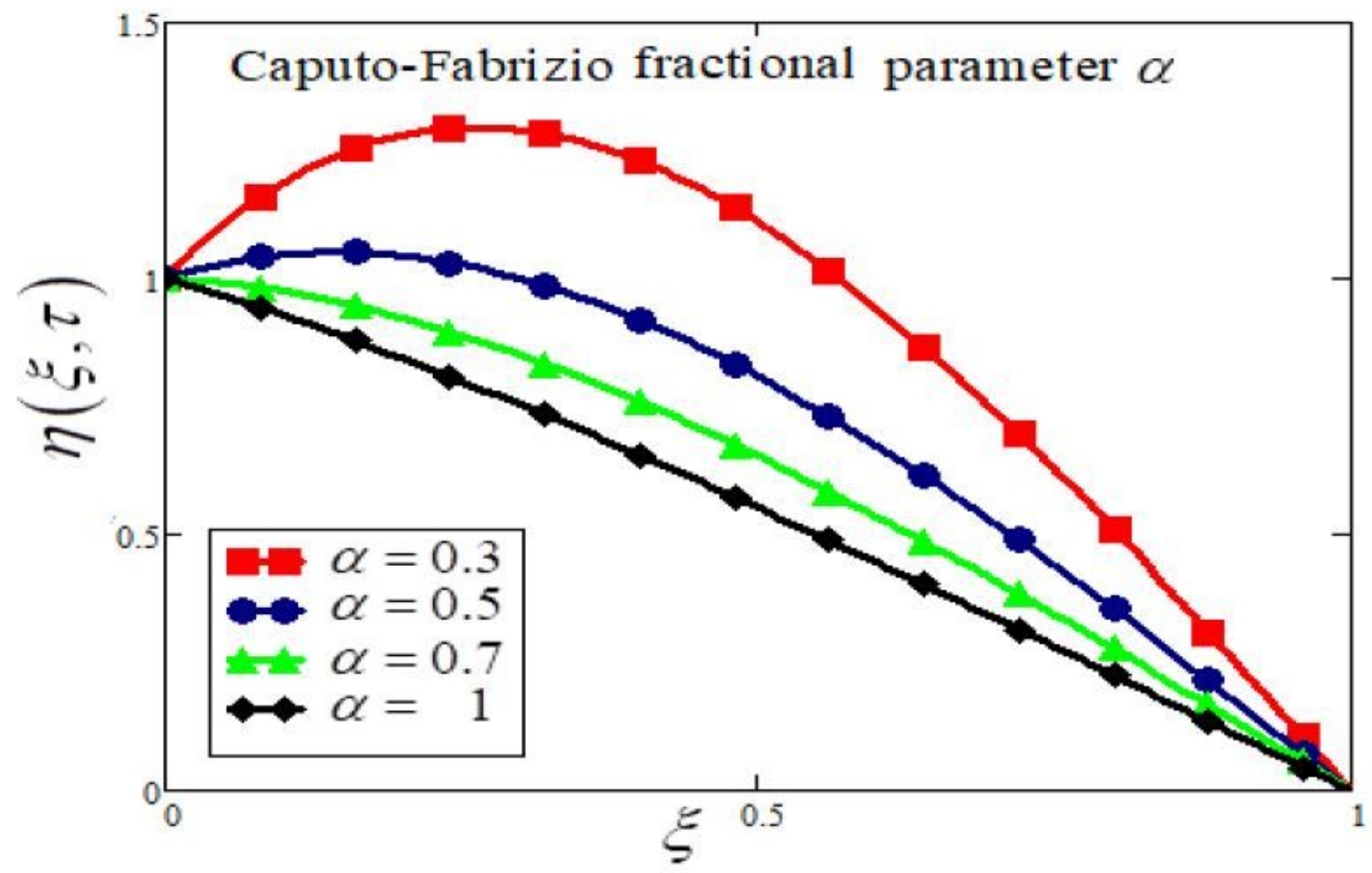

Figure 4

The impact of various values of CF fractional parameter a on CSF velocity when $\lambda=1.2, G=10, \operatorname{Re}=0.6$, $\mathrm{t}=0.6$. 


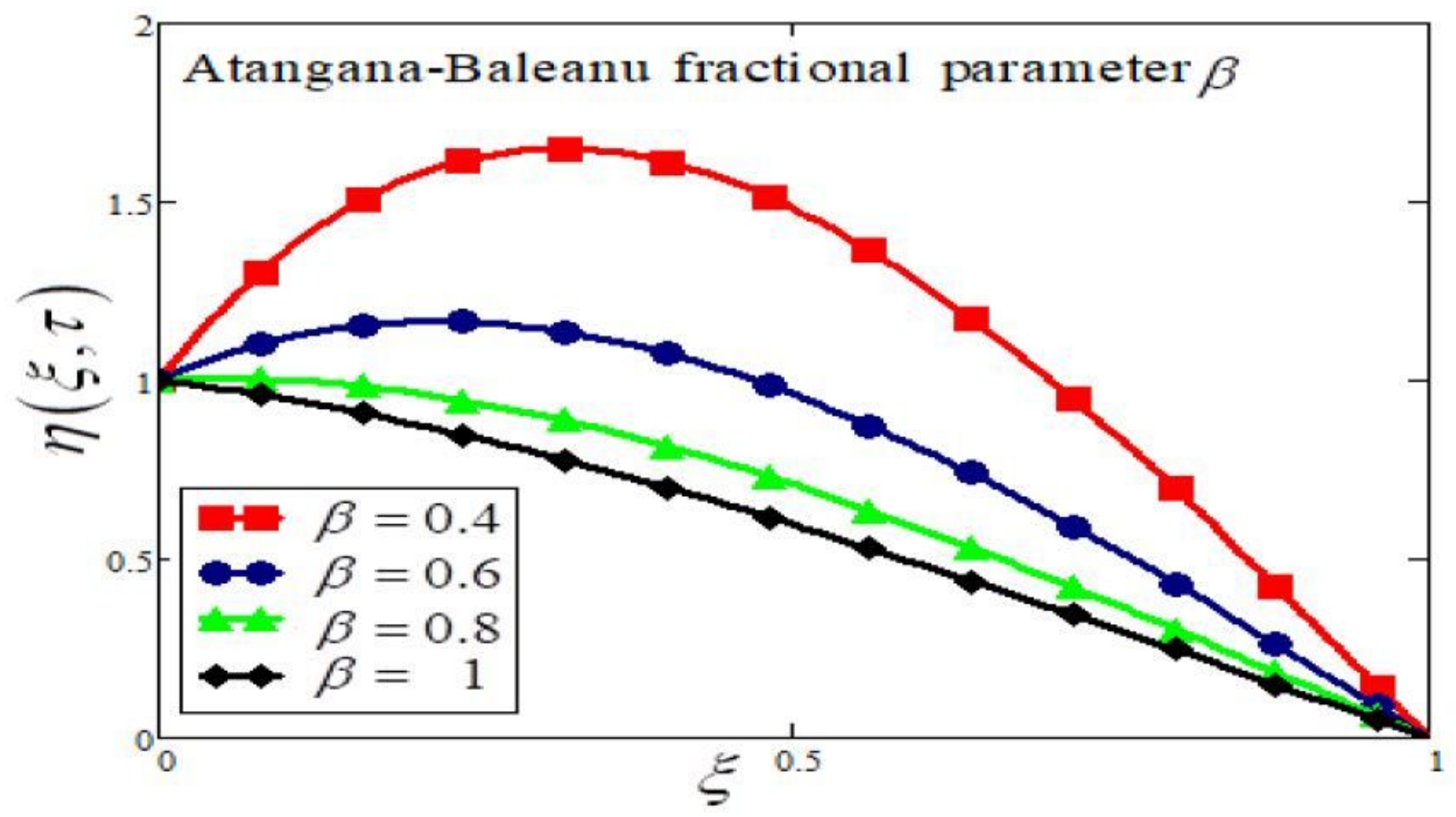

Figure 5

The impact of $A B$ fractional operator on CSF velocity when $\lambda=1.2, G=8, \operatorname{Re}=0.6, \tau=0.8$.
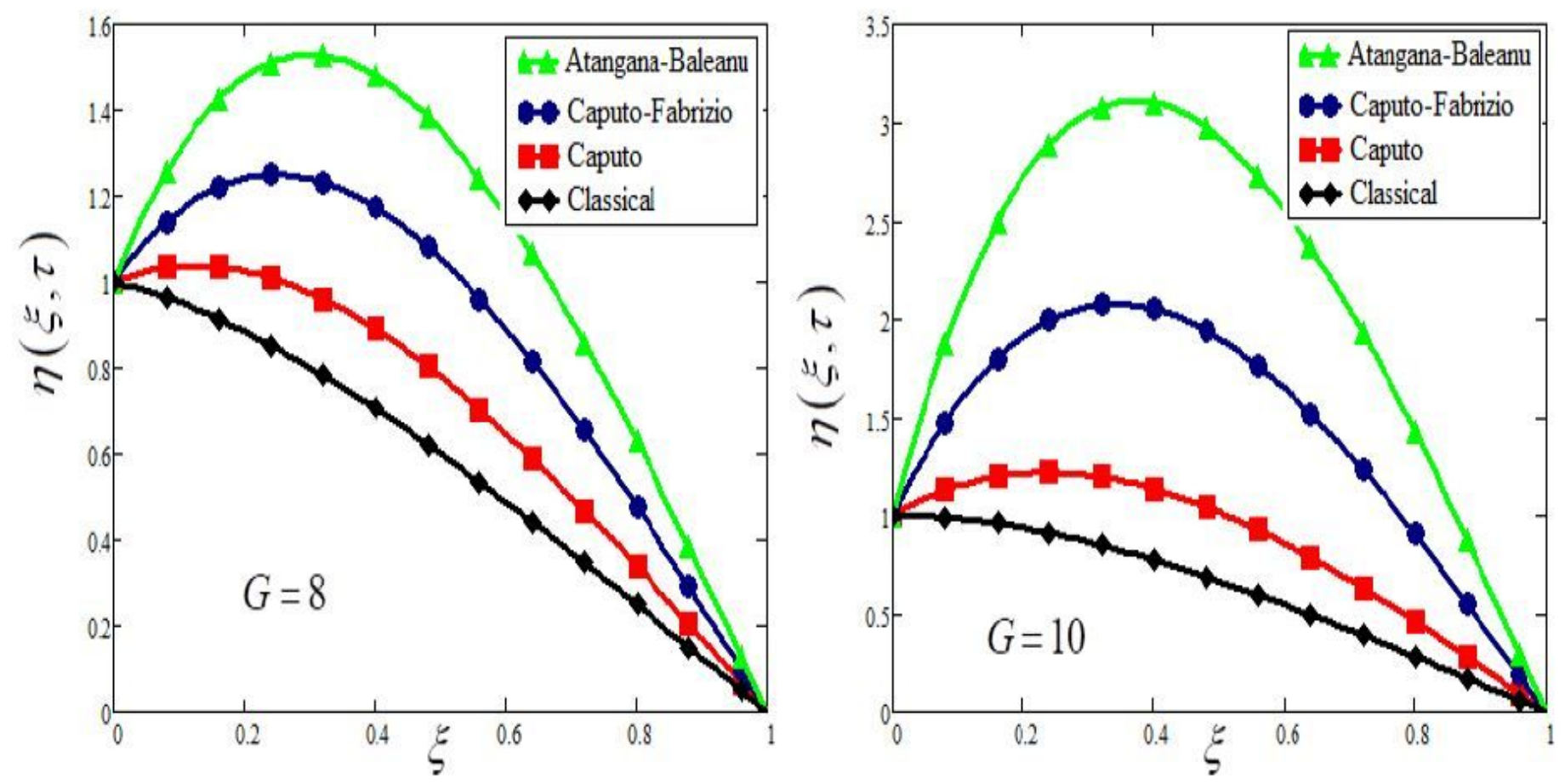

Figure 6 
The impact of external pressure gradient $\mathrm{G}$ on CSF velocity for $\mathrm{C}, \mathrm{CF}, \mathrm{AB}$ and classical velocity when $\operatorname{Re}=0.4, \tau=0.7, \alpha=\beta=\gamma=0.5$ and $\lambda=0.8$.
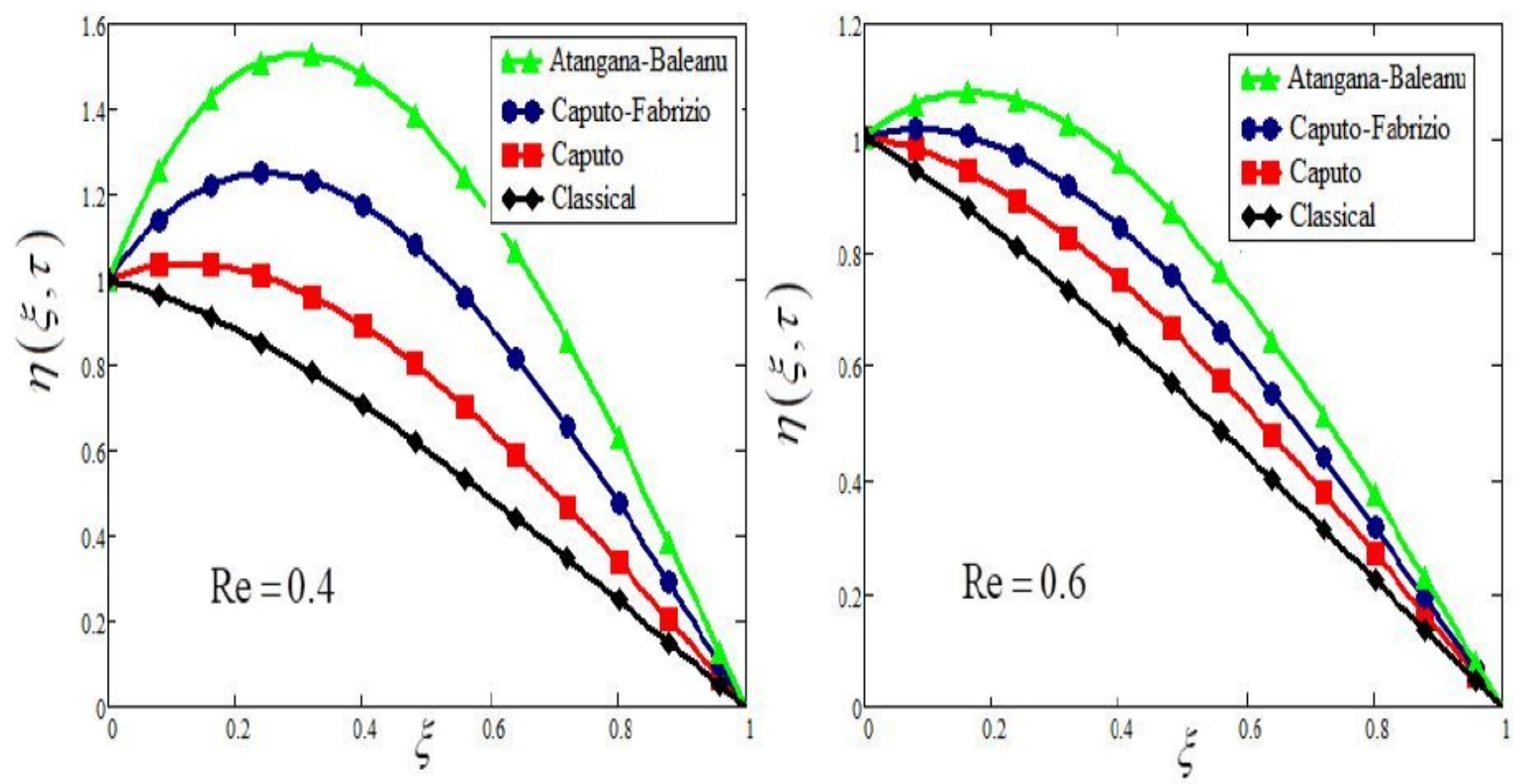

Figure 7

The impact of Reynolds number Re on CSF velocity for $C, C F, A B$ and classical velocity when $\mathrm{G}=8$, $\alpha=\beta=\gamma=0.5, \tau=0.7$ and $\lambda=0.8$. 

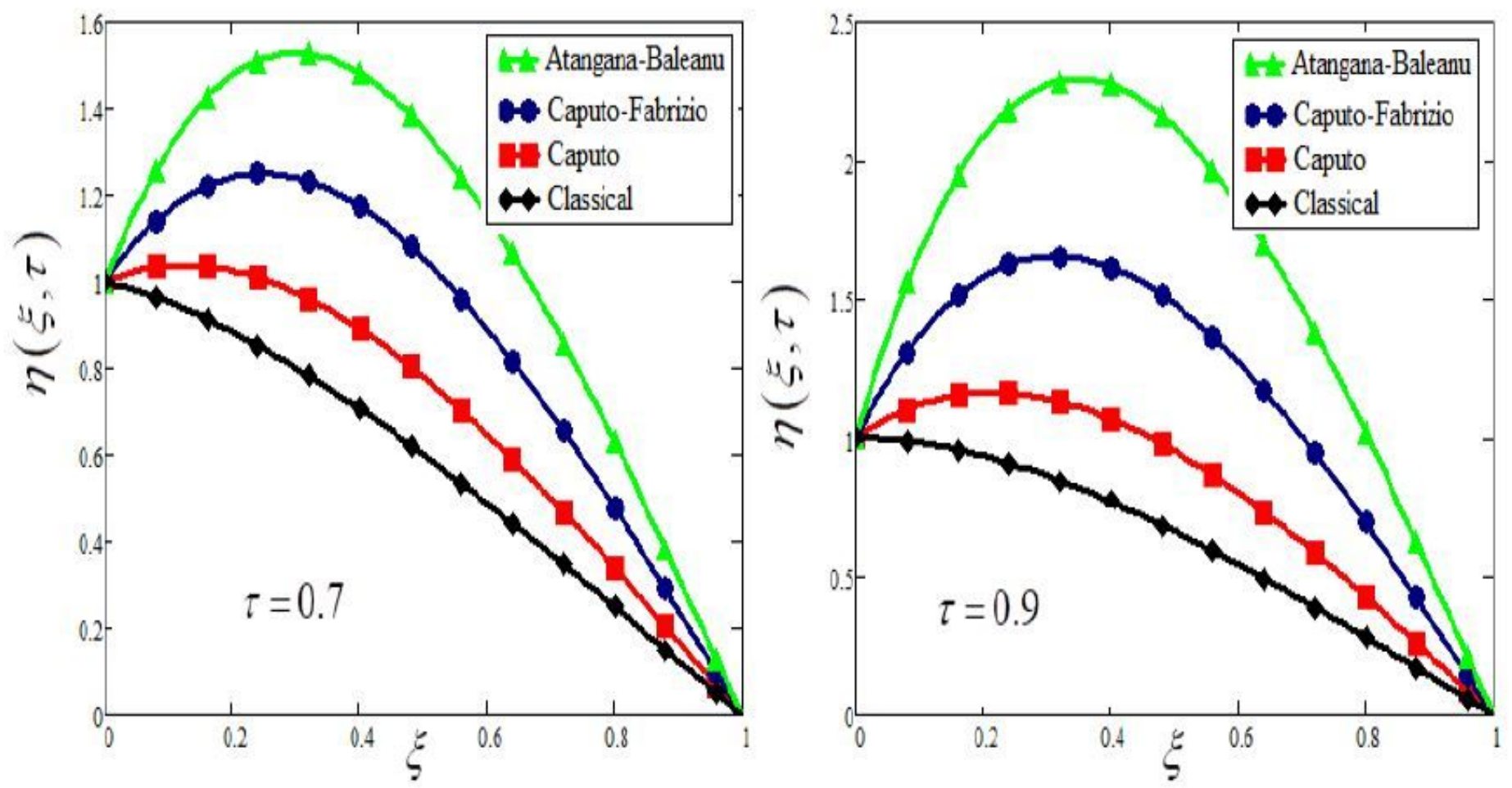

Figure 8

The impact of time $\tau$ on velocity profile for $C, C F, A B$ and classical $C S F$ when $G=8, a=\beta=\gamma=0.5, R e=0.4$ and $\lambda=0.8$.
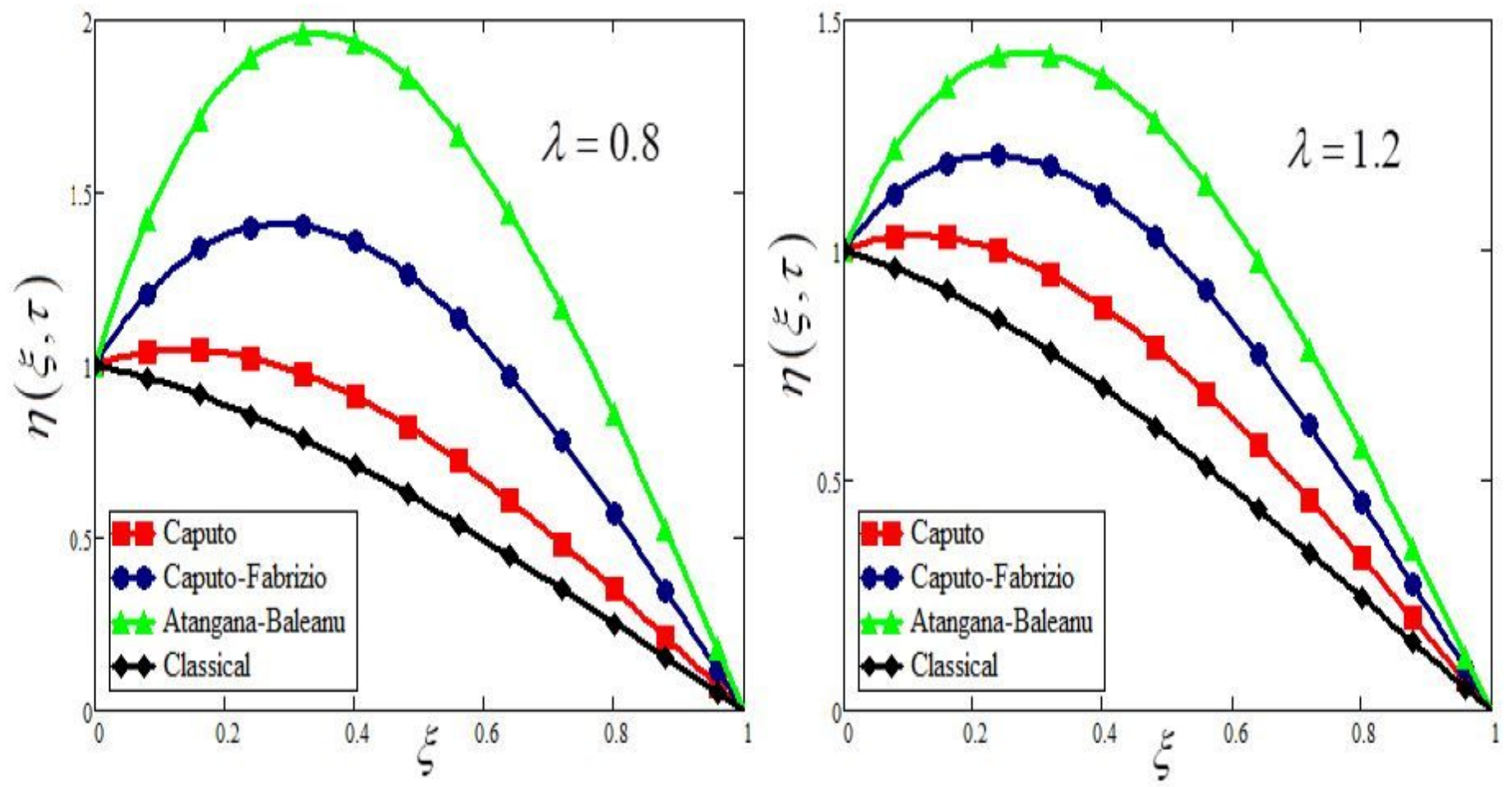
Figure 9

Comparison of CSF velocity profile for $C, C F, A B$ and classical velocity with variation of CSF parameter $\lambda$ when $\mathrm{G}=8, \mathrm{a}=\beta=\gamma=0.5, \tau=0.7$ and $\mathrm{Re}=0.8$.

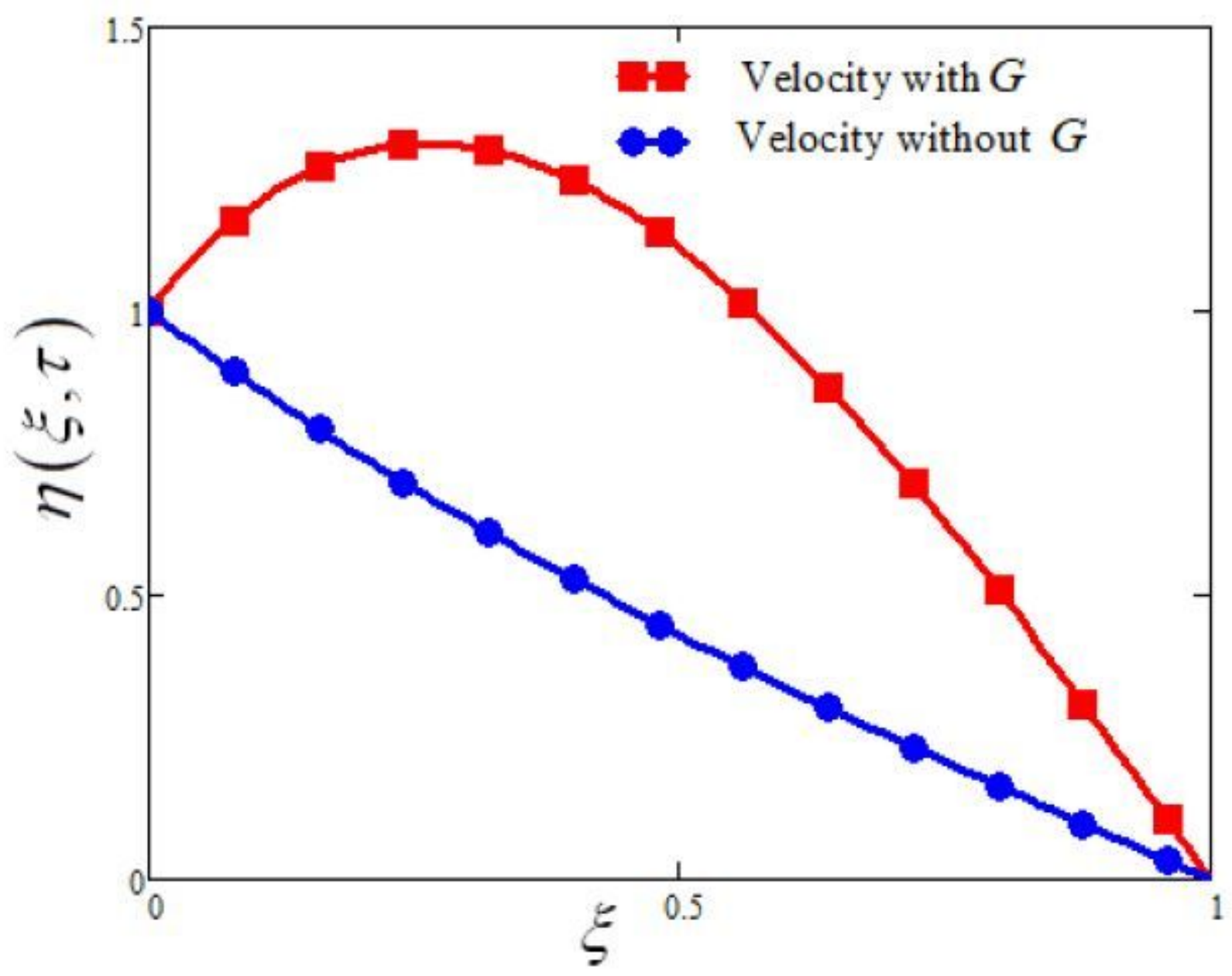

Figure 10

CSF velocity with $G$ and without $G$ when $\operatorname{Re}=0.6, \tau=0.6, a=0.5$ and $\lambda=1.2$ 


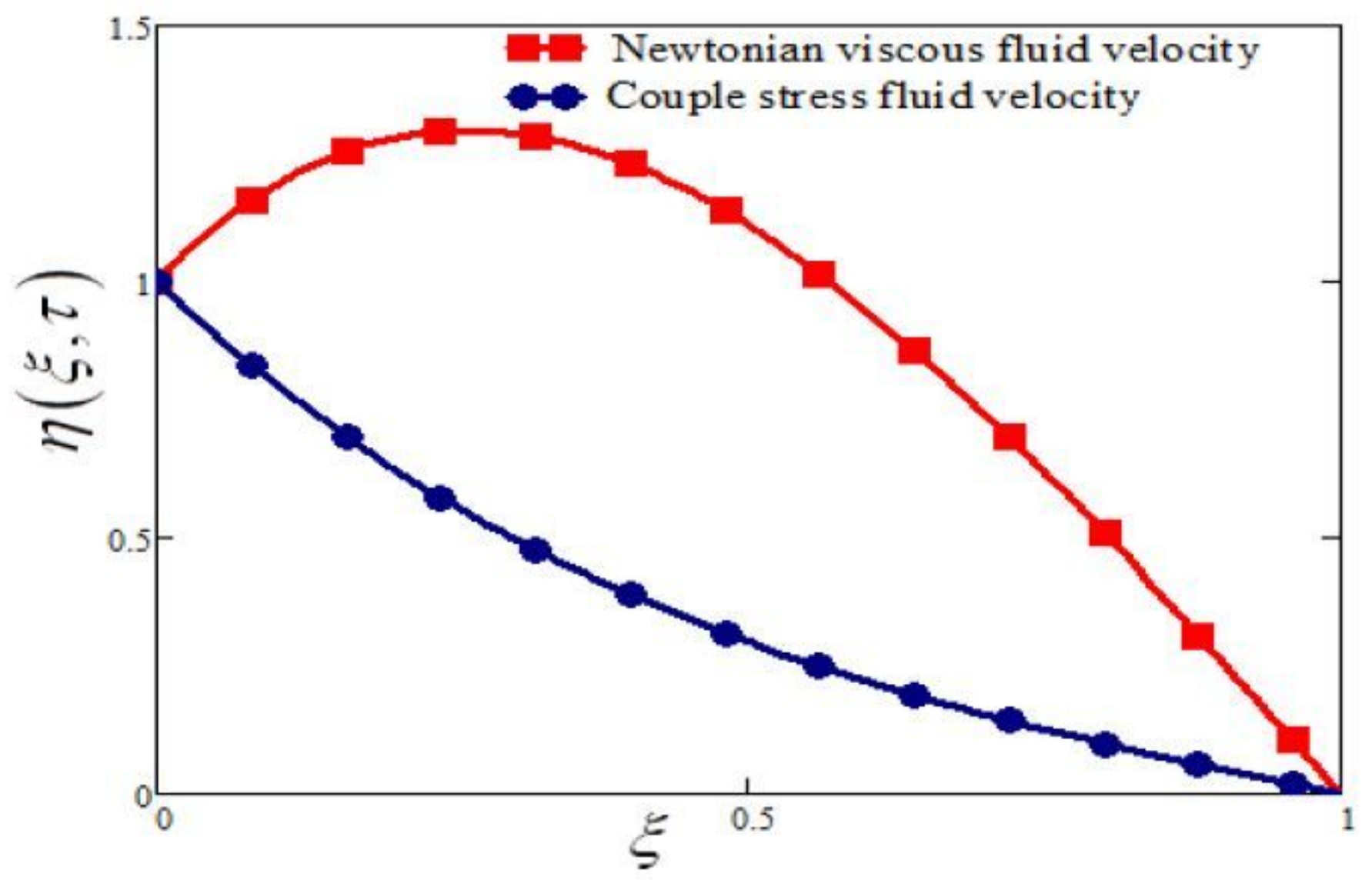

Figure 11

CSF and Newtonian fluid velocity when $\mathrm{G}=8, \mathrm{Re}=0.6$ and $\tau=0.7$. 